\title{
Simultaneous topology and size optimization of a 3D variable-length structure described by the ALE-ANCF
}

Sun, Jialiang; Tian, Qiang; Hu, Haiyan; Pedersen, Niels L.

Published in:

Mechanism and Machine Theory

Link to article, DOI:

10.1016/j.mechmachtheory.2018.07.013

Publication date:

2018

Document Version

Peer reviewed version

Link back to DTU Orbit

Citation (APA):

Sun, J., Tian, Q., Hu, H., \& Pedersen, N. L. (2018). Simultaneous topology and size optimization of a 3D variable-length structure described by the ALE-ANCF. Mechanism and Machine Theory, 129, 80-105. https://doi.org/10.1016/j.mechmachtheory.2018.07.013

\section{General rights}

Copyright and moral rights for the publications made accessible in the public portal are retained by the authors and/or other copyright owners and it is a condition of accessing publications that users recognise and abide by the legal requirements associated with these rights.

- Users may download and print one copy of any publication from the public portal for the purpose of private study or research.

- You may not further distribute the material or use it for any profit-making activity or commercial gain

- You may freely distribute the URL identifying the publication in the public portal 


\title{
Simultaneous topology and size optimization of a 3D variable-length structure described by the ALE-ANCF
}

\author{
Jialiang Sun ${ }^{\mathrm{a}}$, Qiang Tian ${ }^{\mathrm{b}}$, Haiyan $\mathrm{Hu}^{\mathrm{a}, \mathrm{b},{ }^{*}}$, Niels L. Pedersen ${ }^{\mathrm{c}}$ \\ ${ }^{a}$ State Key Laboratory of Mechanics and Control of Mechanical Structures, College of Aerospace \\ Engineering, Nanjing University of Aeronautics and Astronautics, Nanjing 210016, China
}

${ }^{\mathrm{b}}$ MOE Key Laboratory of Dynamics and Control of Flight Vehicle, School of Aerospace Engineering, Beijing Institute of Technology, Beijing 100081, China

${ }^{\mathrm{c}}$ Department of Mechanical Engineering, Solid Mechanics, Technical University of Denmark, Kongens Lyngby 2800, Denmark

\begin{abstract}
An efficient optimization approach is proposed to simultaneously optimize the topology and geometrical sizes of a three-dimensional (3D) variable-length structure. First of all, in a unified global coordinate system, a flexible multibody system (FMBS) model is established to describe the extension and contraction dynamics of a variable-length structure. The variable-length flexible structure is meshed by the proposed arbitrary Lagrangian-Eulerian (ALE) solid elements of absolute nodal coordinate formulation (ANCF). To consider the variable-length behaviors, the classic equivalent static loads (ESL) is redefined by introducing the concept of virtual design domain. With the help of the ESL, the dynamic response optimization of the variable-length structure is turned into a static one. A simultaneous topology and size optimization approach is initially proposed via the moving morphable components (MMC). By appropriately arranging the initial configuration of the components and linking all or part of the design variables of a component with others', the variable-length structure can be designed as a homogeneous or heterogeneous periodic structure. The approach is numerically implemented via an additional constraint that modifies the sensitivities with respect to some design variables. Finally, three numerical examples are presented to demonstrate the effectiveness and efficiency of the proposed optimization approach.
\end{abstract}

Keywords: Topology optimization; Size optimization; Variable-length structure; Flexible multibody system; Equivalent static loads; Moving morphable components

\footnotetext{
* Corresponding author.

E-mail addresses: sunjialiang@nuaa.edu.cn (J. Sun), tianqiang_hust@aliyun.com (Q. Tian), hhyae@nuaa.edu.cn (H. Hu),nlp@mek.dtu.dk (N.L. Pedersen).
} 


\section{Introduction}

Three-dimensional (3D) variable-length structures have been widely used in industries, especially in space engineering for their high packaging ratio and working flexibility. The good examples include the deployable articulated mast systems, the telescoping boom systems, and the deployable solar sail systems [1-4]. For the convenience of compact and efficient stowage, these variable-length structures are usually designed to be a kind of homogeneous periodic structures, that is, the uniform lattice structures at the macro-level $[4,5]$. The homogeneous periodic structure, however, may not be the best choice for the design of a 3D variable-length structure. This is probably due to the fact that the design of the lattice cells is usually deployment oriented rather than load-bearing oriented. In other words, the layout of the bars in one lattice cell is normally placed for the deployable purpose. Besides, the bars in different lattice cells are usually designed exactly the same for the sake of fast manufacturing and assembling. As a consequence, it is important to embed the optimization approaches into the design of these variable-length structures for better performance.

Very few studies have focused on the optimization of the variable-length structures. The major reason is that these variable-length structures may undergo both large overall motions and large deformations during their deployment process. It is a great challenge, hence, to describe the dynamics of a variable-length structure during the optimization process. To account for the sliding joint and the connections with other bodies of a variable-length structure, it is necessary to establish a flexible multibody system (FMBS) model governed by a set of differential algebraic equations (DAEs), rather than a set of ordinary differential equations (ODEs). A few formulations have been available to describe an FMBS with variable-length bodies, such as the variable-domain finite element (VFE) model based on the floating frame of reference formulation (FFRF) [6, 7], the arbitrary Lagrangian-Eulerian (ALE) description based on the absolute nodal coordinate formulation (ANCF) [3, 8, 9], and a nonlinear finite element approach proposed by Geradin and Cardona [10]. As to the ALE-ANCF, several finite elements have been proposed for different purposes [3, 9, 11-14], including the topology optimization of a planar variable-length structure in the work by Sun et al. [3]. Nevertheless, these studies have neither considered the 3D cases nor performed the optimization design for the variable-length periodic structures [3].

The optimization of a 3D variable-length structure can be performed either directly from the aforementioned dynamic analysis of FMBS or from a series of equivalent static analyses $[15,16]$. The former is called the fully coupled method or the integrated optimization method [16-18], while the latter is called the weakly coupled method or the equivalent static loads (ESL) method [16, 19-25]. Compared with the fully coupled method, the weakly coupled method has two significant merits. Firstly, it is simple to implement by replacing the dynamic response simulation of FMBS with static responses simulation at each time step $[15,16]$. Secondly, the expensive and timeconsuming dynamic analysis and sensitivity analysis of FMBS can be omitted in the inner optimization iteration [3, 20, 25]. During each outer optimization cycle, therefore, the dynamic analysis of FMBS only needs to be carried out once to obtain the ESL sets. 
However, it is difficult to derive the ESL sets from the dynamic analysis of FMBS due to the variable-length characteristics. Sun et al. [3] initially proposed the concepts of actual and virtual design domains to redefine the ESL sets for the variable-length structure. According to their work [3], a virtual design domain is defined as the unstretched domain for the extension case, and the contracting domain for the contraction case of a variable-length structure. An actual design domain is the stretching domain for the extension case, and the uncontracted domain for the contraction case. Obviously, the two domains are time-varying, but the whole design domain defined as the union of the two domains is time-invariant.

With the help of the ESL, the dynamic response optimization of a $3 \mathrm{D}$ variablelength structure can be transformed into a static one [16, 19-25]. After performing optimization, the variable-length structure can be designed as a kind of periodic structure or the lattice structure at the macro-level. As a matter of fact, recent years have witnessed an increased number of studies on the structural optimization of the lattice structures. For example, Reinhart and Teufelhart optimized the periodically arranged lattice structures by adapting the struts to the flux of force to avoid the unfavorably loaded shear forces [26]. Gu et al. introduced the topology optimization into the design of the lattice structure configuration for rapid casting patterns [27]. Stankovic et al. performed the optimization of the multimaterial mesoscale lattice structures under a given volume by using a generalized optimal criteria method [28]. Tang et al. proposed a new design method for the periodic lattice structures in order to optimize the thickness distribution of the lattice struts with the help of the bidirectional evolutionary structural optimization [5]. Zhang et al. designed a variable-density cellular lattice structure by optimizing the density distribution of a solid material block and remapping the material density to a hexagon cell with a circular hole [29]. Liu et al. proposed a design approach for graded lattice structures by using the moving morphable components/voids (MMC/MMV)-based topology optimization method [30]. Wu et al. optimized the lattice structures by using the density-based topology optimization method at two length scales, i.e., mesoscale and macroscale [31]. Wang et al. developed a novel design method that could jointly optimize the geometries of a global structure, lattice mesostructures and the global distributions of mesostructures, and also verified it through experiment tests [32, 33]. It is easy to track more studies in [5, 28-31, 34-36]. However, most previous studies focused on the optimization of a 2D lattice structure at the micro- or meso-level [5, 29, 30, 34-36], and separated the structure topology optimization and the size optimization of the lattice cells [28, 29, 31].

In this study, a new simultaneous topology and size optimization approach is proposed to design a 3D variable-length structure via the MMC method initially proposed by Guo et al. [37, 38] and Zhang et al. [39, 40]. This approach not only maintains the merits of a periodic lattice structure at the macro-level but can also simultaneously optimize the topology and thicknesses of those lattice cells. For the dynamic analysis of a 3D variable-length structure, a kind of axially variable-length solid element of ALE-ANCF is also developed to establish the FMBS model. Based on the authors' previous work [3], the classic ESL sets are redefined to treat the variablelength behaviors of the structure. To solve the static optimization problem, firstly the 
3D variable-length structure to be optimized is divided into several lattice cells and occupied with the same initial configuration of the components in each lattice cell. Then, the $3 \mathrm{D}$ variable-length structure can be designed as a homogeneous or a heterogeneous periodic structure by introducing an additional constraint. The constraint modifies the sensitivities with respect to the linked design variables of the components in different lattice cells to be identical and always equal to the average value of their original sensitivities during the optimization iteration. The proposed optimization approach can efficiently optimize a 3D variable-length structure because the introduction of the ESL and the MMC can dramatically reduce the number of dynamic analysis of FMBS and the number of the design variables for 3D cases [23, 39].

The paper is organized as follows. In Section 2, the basics of the variable-length solid element of ALE-ANCF are described and an FMBS model is established for a 3D variable-length structure. In Section 3, the weakly coupled optimization formulation of the variable-length structure is outlined and the ESL sets for the variable-length structure are redefined. Then, the ESL-based optimization computation flow is briefly given. In Section 4, the fundamentals of the 3D MMC are revisited and the detailed computation process of the simultaneous topology and size optimization approach is presented. In Section 5, three numerical examples are provided to validate the proposed optimization approach. Finally, some concluding remarks are drawn in Section 6.

\section{FMBS model for a 3D variable-length structure}

In this section, a variable-length solid element of ALE-ANCF is developed to establish an FMBS model for a 3D variable-length structure. The corresponding kinematic constraints like a sliding joint during the deployment are presented via the method of Lagrangian multipliers.

\subsection{Kinematic equations of a solid element of ALE-ANCF}

In the framework of the ALE-ANCF, as shown in Fig. 1, the configuration of a solid element with a variable length in $z$-direction can be described by the nodal position vectors $\mathbf{r}_{i}(i=1, \cdots, 8)$, the nodal slope vectors $\mathbf{r}_{i, x}=\partial \mathbf{r}_{i} / \partial x, \quad \mathbf{r}_{i, y}=\partial \mathbf{r}_{i} / \partial y$ and $\mathbf{r}_{i, z}=\partial \mathbf{r}_{i} / \partial z \quad(i=1, \cdots, 8)$, and two material coordinates $h_{1}, h_{2}$. The whole 98 generalized coordinates of the solid element are then given by

$$
\mathbf{q}=\left[\begin{array}{lllllllllll}
\mathbf{r}_{1}^{\mathrm{T}} & \mathbf{r}_{1, x}^{\mathrm{T}} & \mathbf{r}_{1, y}^{\mathrm{T}} & \mathbf{r}_{1, z}^{\mathrm{T}} & \cdots & \mathbf{r}_{8}^{\mathrm{T}} & \mathbf{r}_{8, x}^{\mathrm{T}} & \mathbf{r}_{8, y}^{\mathrm{T}} & \mathbf{r}_{8, z}^{\mathrm{T}} & h_{1} & h_{2}
\end{array}\right]^{\mathrm{T}},
$$

where $h_{1}$ and $h_{2}$ are measured along the $z$-direction of the reference configuration from the head face. The head face is defined as one of the cross-sections of the undeformed variable-length structure, in which the material coordinate $h=0$. In the ALE framework, the nodes of the solid element are not fixed to any material points and can slide freely along the structure. If $\dot{h}_{2}-\dot{h}_{1}>0$, the length of the solid element in the $z$-direction increases. Otherwise, it decreases or keeps constant. When the length varies, mass flows 
in or out of the solid element, as a result of which, the solid element has a mass-varying property.

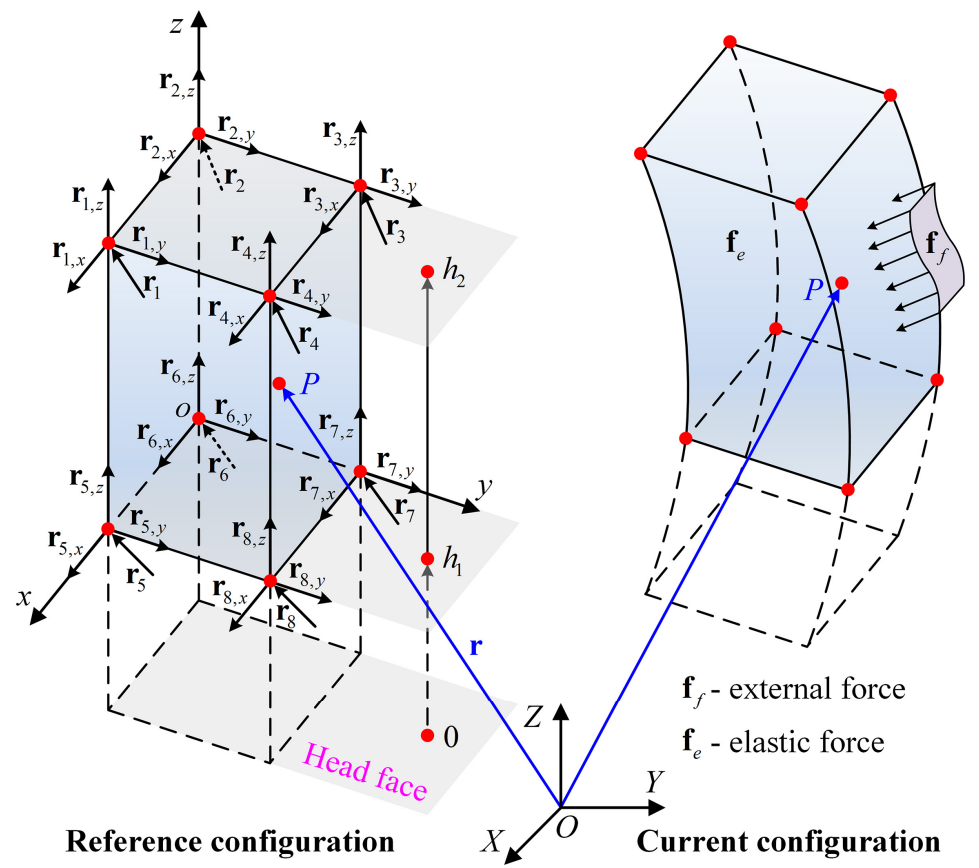

Fig. 1. An axially variable-length solid element of ALE-ANCF.

Based on the local coordinates $(x, y, z)$, the position vector $\mathbf{r}$ of an arbitrary point $P$ in the global frame of coordinates $O-X Y Z$ can be defined by the Hermite interpolation as follows

$$
\mathbf{r}(x, y, z)=\mathbf{N}_{e}(x, y, z) \mathbf{q}_{e},
$$

where

$$
\begin{aligned}
\mathbf{q}_{e} & =\left[\begin{array}{lllllllll}
\mathbf{r}_{1}^{\mathrm{T}} & \mathbf{r}_{1, x}^{\mathrm{T}} & \mathbf{r}_{1, y}^{\mathrm{T}} & \mathbf{r}_{1, z}^{\mathrm{T}} & \cdots & \mathbf{r}_{8}^{\mathrm{T}} & \mathbf{r}_{8, x}^{\mathrm{T}} & \mathbf{r}_{8, y}^{\mathrm{T}} & \mathbf{r}_{8, z}^{\mathrm{T}}
\end{array}\right]^{\mathrm{T}}, \\
\mathbf{N}_{e} & =\left[\begin{array}{lllllllll}
N_{1,0} \mathbf{I} & N_{1,1} \mathbf{I} & N_{1,2} \mathbf{I} & N_{1,3} \mathbf{I} & \cdots & N_{8,0} \mathbf{I} & N_{8,1} \mathbf{I} & N_{8,2} \mathbf{I} & N_{8,3} \mathbf{I}
\end{array}\right],
\end{aligned}
$$

with I denoting the $3 \times 3$ identity matrix and the entries of $\mathbf{N}_{e}$ defined as follows

$$
\begin{aligned}
& N_{i, 0}=\frac{1}{16}(1 \pm \xi)(1 \pm \eta)(1 \pm \zeta)\left(2 \pm \xi \pm \eta \pm \zeta-\xi^{2}-\eta^{2}-\zeta^{2}\right), \\
& N_{i, 1}= \pm \frac{1}{32} a\left(\xi^{2}-1\right)(1 \pm \xi)(1 \pm \eta)(1 \pm \zeta), \\
& N_{i, 2}= \pm \frac{1}{32} b\left(\eta^{2}-1\right)(1 \pm \xi)(1 \pm \eta)(1 \pm \zeta), \\
& N_{i, 3}= \pm \frac{1}{32} c\left(\zeta^{2}-1\right)(1 \pm \xi)(1 \pm \eta)(1 \pm \zeta) .
\end{aligned}
$$

Here, $i=1, \cdots, 8$ and the detailed expressions of Eq. (5) are listed in the "Appendix". $a, b$, and $c=h_{2}-h_{1}$ are the undeformed lengths of the element reference configuration in $x$-, $y$ - and $z$-directions and

$$
\xi=\frac{x-a / 2}{a / 2}, \eta=\frac{y-b / 2}{b / 2}, \zeta=\frac{z-c / 2}{c / 2}=\frac{2 h-h_{1}-h_{2}}{h_{2}-h_{1}} .
$$


According to Eq. (2), the vectors of velocity and acceleration of the point $P$ in the solid element can be directly derived by using time differentiation as follows

$$
\dot{\mathbf{r}}=\mathbf{N} \dot{\mathbf{q}}, \ddot{\mathbf{r}}=\mathbf{N} \ddot{\mathbf{q}}+\ddot{\mathbf{r}}_{h},
$$

where

$$
\begin{aligned}
& \mathbf{N}=\left[\begin{array}{lll}
\mathbf{N}_{e} & \mathbf{N}_{e, h_{1}} \mathbf{q}_{e} & \mathbf{N}_{e, h_{2}} \mathbf{q}_{e}
\end{array}\right], \\
& \ddot{\mathbf{r}}_{h}=2\left(\mathbf{N}_{e, h_{1}} \dot{h}_{1}+\mathbf{N}_{e, h_{2}} \dot{h}_{2}\right) \dot{\mathbf{q}}_{e}+\left(\mathbf{N}_{e, h_{1} h_{1}} \dot{h}_{1}^{2}+2 \mathbf{N}_{e, h_{1} h_{2}} \dot{h}_{1} \dot{h}_{2}+\mathbf{N}_{e, h_{2} h_{2}} \dot{h}_{2}^{2}\right) \mathbf{q}_{e},
\end{aligned}
$$

and $\dot{\mathbf{q}}=\left[\begin{array}{lll}\dot{\mathbf{q}}_{e}^{\mathrm{T}} & \dot{h}_{1} & \dot{h}_{2}\end{array}\right]^{\mathrm{T}}$ is the vector of generalized velocity of the solid element.

\subsection{Dynamic equation of a solid element of $A L E-A N C F$}

As the solid element of ALE-ANCF has a property of mass-varying due to the variable length, the Lagrange equations cannot be directly used to establish the dynamic equation, but the D'Alembert's principle can [41]. According to the D'Alembert's principle, the total virtual work of the impressed forces including external force $\mathbf{f}_{f}$ and elastic force $\mathbf{f}_{e}$ plus the inertial force vanishes for an arbitrary reversible displacement, i.e.,

$$
\int_{V_{0}} \delta \mathbf{r}^{\mathrm{T}}\left(\mathbf{f}_{f}+\mathbf{f}_{e}-\rho \ddot{\mathbf{r}}\right) \mathrm{d} V=0,
$$

where $V_{0}$ is the initial volume of the solid element.

As the virtual displacement reads

$$
\delta \mathbf{r}=\mathbf{N}_{e} \delta \mathbf{q}_{e}+\delta \mathbf{N}_{e} \mathbf{q}_{e}=\mathbf{N} \delta \mathbf{q},
$$

the virtual work of the forces in Eq. (10) yields

$$
\begin{aligned}
& \int_{V_{0}} \delta \mathbf{r}^{\mathrm{T}} \mathbf{f}_{f} \mathrm{~d} V=\delta \mathbf{q}^{\mathrm{T}} \int_{V_{0}} \mathbf{N}^{\mathrm{T}} \mathbf{f}_{f} \mathrm{~d} V=\delta \mathbf{q}^{\mathrm{T}} \frac{1}{8} a b\left(h_{2}-h_{1}\right) \int_{-1}^{1} \int_{-1}^{1} \int_{-1}^{1} \mathbf{N}^{\mathrm{T}} \mathbf{f}_{f} \mathrm{~d} \xi \mathrm{d} \eta \mathrm{d} \zeta, \\
& \int_{V_{0}} \delta \mathbf{r}^{\mathrm{T}} \mathbf{f}_{e} \mathrm{~d} V=-\delta \mathbf{q}^{\mathrm{T}} \frac{1}{8} a b\left(h_{2}-h_{1}\right) \int_{-1}^{1} \int_{-1}^{1} \int_{-1}^{1}\left(\frac{\partial \boldsymbol{\varepsilon}}{\partial \mathbf{q}}\right)^{\mathrm{T}} \mathbf{E} \boldsymbol{\varepsilon} \mathrm{d} \xi \mathrm{d} \eta \mathrm{d} \zeta, \\
& \int_{V_{0}} \delta \mathbf{r}^{\mathrm{T}}(-\rho \ddot{\mathbf{r}}) \mathrm{d} V=-\delta \mathbf{q}^{\mathrm{T}} \frac{1}{8} a b\left(h_{2}-h_{1}\right) \int_{-1}^{1} \int_{-1}^{1} \int_{-1}^{1}\left(\rho \mathbf{N}^{\mathrm{T}} \mathbf{N} \ddot{\mathbf{q}}+\rho \mathbf{N}^{\mathrm{T}} \ddot{\mathbf{r}_{h}}\right) \mathrm{d} \xi \mathrm{d} \eta \mathrm{d} \zeta .
\end{aligned}
$$

Here, $\rho$ is the mass density of the solid element. The Green-Lagrange strain tensor $\boldsymbol{\varepsilon}$ and the elastic coefficient matrix $\mathbf{E}$ are as follows

$$
\boldsymbol{\varepsilon}=\left[\begin{array}{c}
\frac{\mathbf{r}_{,}^{\mathrm{T}} \mathrm{r}_{x, x}-1}{2} \\
\frac{\mathbf{r}_{, y}^{\mathrm{T}} \mathbf{r}_{y,}-1}{2} \\
\frac{r_{, h}^{\mathrm{T}} \mathbf{r}_{h,}-1}{2} \\
\mathbf{r}_{, x}^{\mathrm{T}} \mathbf{r}_{, y} \\
\mathbf{r}_{, y}^{\mathrm{T}} \mathbf{r}_{, h} \\
\mathbf{r}_{, h}^{\mathrm{T}} \mathbf{r}_{, x}
\end{array}\right], \mathbf{E}=\frac{E}{(1+v)(1-2 v)}\left[\begin{array}{cccccc}
1-v & v & v & 0 & 0 & 0 \\
v & 1-v & v & 0 & 0 & 0 \\
v & v & 1-v & 0 & 0 & 0 \\
0 & 0 & 0 & \frac{1-2 v}{2} & 0 & 0 \\
0 & 0 & 0 & 0 & \frac{1-2 v}{2} & 0 \\
0 & 0 & 0 & 0 & 0 & \frac{1-2 v}{2}
\end{array}\right],
$$

with $E$ and $v$ denoting Young's modulus and Poisson's ratio, respectively for the material of the finite element. 
Considering the kinematic constraints $\boldsymbol{\Phi}^{e}(\mathbf{q}, t)=\mathbf{0}$, the dynamic equation of the solid element can be formulated via the method of Lagrangian multipliers as follows

$$
\begin{aligned}
& \mathbf{M}^{e} \ddot{\mathbf{q}}+\mathbf{F}_{a}^{e}+\mathbf{F}_{e}^{e}+\mathbf{\Phi}_{, \mathbf{q}}^{e \mathrm{~T}} \lambda^{e}=\mathbf{F}_{f}^{e}, \\
& \mathbf{M}^{e}=\frac{1}{8} a b\left(h_{2}-h_{1}\right) \int_{-1}^{1} \int_{-1}^{1} \int_{-1}^{1} \rho \mathbf{N}^{\mathrm{T}} \mathbf{N} \mathrm{d} \xi \mathrm{d} \eta \mathrm{d} \zeta, \\
& \mathbf{F}_{a}^{e}=\frac{1}{8} a b\left(h_{2}-h_{1}\right) \int_{-1}^{1} \int_{-1}^{1} \int_{-1}^{1} \rho \mathbf{N}^{\mathrm{T}} \ddot{\mathbf{r}}_{h} \mathrm{~d} \xi \mathrm{d} \eta \mathrm{d} \zeta, \\
& \mathbf{F}_{e}^{e}=\frac{1}{8} a b\left(h_{2}-h_{1}\right) \int_{-1}^{1} \int_{-1}^{1} \int_{-1}^{1}\left(\frac{\partial \boldsymbol{\varepsilon}}{\partial \mathbf{q}}\right)^{\mathrm{T}} \mathbf{E} \boldsymbol{\varepsilon} \mathrm{d} \xi \mathrm{d} \eta \mathrm{d} \zeta, \\
& \mathbf{F}_{f}^{e}=\frac{1}{8} a b\left(h_{2}-h_{1}\right) \int_{-1}^{1} \int_{-1}^{1} \int_{-1}^{1} \mathbf{N}^{\mathrm{T}} \mathbf{f}_{f} \mathrm{~d} \xi \mathrm{d} \eta \mathrm{d} \zeta,
\end{aligned}
$$

where $\mathbf{M}^{e}$ is the time-varying mass matrix of the solid element, $\mathbf{F}_{a}^{e}$ is the vector of generalized additional inertial forces, $\mathbf{F}_{e}^{e}$ is the vector of generalized elastic forces, $\mathbf{F}_{f}^{e}$ is the vector of the generalized external forces, $\lambda^{e}$ is the vector of Lagrangian multipliers and $\boldsymbol{\Phi}_{, \mathbf{q}}^{e}{ }^{\mathrm{T}} \lambda^{e}$ is the vector of generalized constraint forces of the solid element.

\subsection{Dynamic equation of an FMBS}

The dynamic equation of an FMBS modeled via the ALE-ANCF can be expressed as the following set of DAEs with index $3[3,11]$

$$
\left\{\begin{array}{l}
\mathbf{M}(t) \ddot{\mathbf{q}}_{D}+\boldsymbol{\Phi}_{, \mathbf{q}_{D}}^{\mathrm{T}} \lambda=\mathbf{Q}\left(\dot{\mathbf{q}}_{D}, \mathbf{q}_{D}, t\right) \\
\mathbf{\Phi}\left(\mathbf{q}_{D}, t\right)=\mathbf{0}
\end{array}\right.
$$

where $\mathbf{M}(t)$ is the time-varying mass matrix of the system, $\mathbf{q}_{D}$ is the vector of generalized coordinates of the system, $\lambda$ is the vector of Lagrange multipliers.

In the first equation of Eq. (21), $\mathbf{M}(t) \ddot{\mathbf{q}}_{D}$ represents the vector of inertial forces, $\boldsymbol{\Phi}_{, \mathbf{q}_{D}}^{\mathrm{T}} \lambda$ denotes the vector of generalized forces produced by the constraints with $\boldsymbol{\Phi}_{, \mathbf{q}_{D}}=\partial \Phi / \partial \mathbf{q}_{D}$, and $\mathbf{Q}\left(\dot{\mathbf{q}}_{D}, \mathbf{q}_{D}, t\right)$ is the vector of generalized forces which can be computed as follows

$$
\mathbf{Q}\left(\dot{\mathbf{q}}_{D}, \mathbf{q}_{D}, t\right)=\mathbf{F}_{f}\left(\dot{\mathbf{q}}_{D}, \mathbf{q}_{D}, t\right)-\mathbf{F}_{a}\left(\dot{\mathbf{q}}_{D}, \mathbf{q}_{D}, t\right)-\mathbf{F}_{e}\left(\mathbf{q}_{D}, t\right)
$$

Here, $\mathbf{F}_{f}, \mathbf{F}_{a}$ and $\mathbf{F}_{e}$ denote the vectors of generalized external forces, generalized additional inertial forces produced by the variable-length-induced mass flow, and generalized elastic forces, respectively. $\mathbf{M}, \mathbf{F}_{f}, \mathbf{F}_{a}$ and $\mathbf{F}_{e}$ can be assembled from Eqs. (17)-(20). The second equation in Eq. (21) denotes the kinematic constraints.

\subsection{Time integration schemes}


To solve the dynamic equation of an FMBS as shown in Eq. (21), the generalized$\alpha$ method [42-44] is employed. According to the work by Leontiev [45] and Tamma et al. [46], the generalized- $\alpha$ method proposed in 1993 [42] is the same as the three parameters optimal schemes initially proposed by Shao in 1987 [47] and by Shao and Cai in 1988 [48].

The time integration schemes of the generalized- $\alpha$ algorithm can be expressed as

$$
\begin{aligned}
& \mathbf{q}_{D}^{(n+1)}=\mathbf{q}_{D}^{(n)}+\Delta t \dot{\mathbf{q}}_{D}^{(n)}+\Delta t^{2}(0.5-\beta) \mathbf{a}^{(n)}+\Delta t^{2} \beta \mathbf{a}^{(n+1)}, \\
& \dot{\mathbf{q}}_{D}^{(n+1)}=\dot{\mathbf{q}}_{D}^{(n)}+\Delta t(1-\gamma) \mathbf{a}^{(n)}+\Delta t \gamma \mathbf{a}^{(n+1)},
\end{aligned}
$$

where $\Delta t$ is the time step size, the superscript $n$ denotes the $n$-th iteration and $\mathbf{a}$ is an auxiliary vector which can be determined by

$$
\left(1-\alpha_{m}\right) \mathbf{a}^{(n+1)}+\alpha_{m} \mathbf{a}^{(n)}=\left(1-\alpha_{f}\right) \ddot{\mathbf{q}}_{D}^{(n+1)}+\alpha_{f} \ddot{\mathbf{q}}_{D}^{(n)}, \mathbf{a}^{(0)}=\ddot{\mathbf{q}}_{D}^{(0)} .
$$

To ensure second-order accuracy and stability properties, the four parameters $\alpha_{m}$, $\alpha_{f}, \beta$ and $\gamma$ in Eqs. (23)-(25) are chosen according to Shao and Cai [48] as

$$
\alpha_{m}=\frac{2 \rho_{\infty}-1}{\rho_{\infty}+1}, \alpha_{f}=\frac{\rho_{\infty}}{\rho_{\infty}+1}, \beta=\frac{1}{\left(\rho_{\infty}+1\right)^{2}}, \gamma=\frac{1}{2}-\alpha_{m}+\alpha_{f},
$$

where $\rho_{\infty} \in[0,1]$ is a controllable parameter that controls the numerical dissipation in the high-frequency range so as to well preserve the low-frequency responses and damp the high-frequency responses. Here, $\rho_{\infty}$ is chosen to be 0.8 for all numerical examples.

To solve the dynamic equation (21) at time step $t^{(n+1)}$, based on the work by Arnold and Brüls [43] and Tromme et al. [17], the responses can be computed by

$$
\begin{gathered}
\mathbf{q}_{D}^{(n+1)}=\mathbf{q}_{D}^{(n+1)}+\Delta \mathbf{q}_{D}, \quad \dot{\mathbf{q}}_{D}^{(n+1)}=\dot{\mathbf{q}}_{D}^{(n+1)}+\hat{\gamma} \Delta \mathbf{q}_{D}, \\
\ddot{\mathbf{q}}_{D}^{(n+1)}=\ddot{\mathbf{q}}_{D}^{(n+1)}+\hat{\beta} \Delta \mathbf{q}_{D}, \lambda^{(n+1)}=\boldsymbol{\lambda}^{(n+1)}+\Delta \boldsymbol{\lambda},
\end{gathered}
$$

where $\hat{\beta}=\frac{1-\alpha_{m}}{\Delta t^{2} \beta\left(1-\alpha_{f}\right)}$ and $\hat{\gamma}=\frac{\gamma}{\Delta t \beta}$ are defined to ensure that $\partial \ddot{\mathbf{q}}_{D} / \partial \mathbf{q}_{D}=\hat{\beta} \mathbf{I}$ and $\partial \dot{\mathbf{q}}_{D} / \partial \mathbf{q}_{D}=\hat{\gamma} \mathbf{I}$. The corrections $\Delta \mathbf{q}_{D}$ and $\Delta \lambda$ in Eq. (27) are obtained from

$$
\left[\begin{array}{cc}
\Delta \mathbf{q}_{D}^{\mathrm{T}} & \Delta \boldsymbol{\lambda}^{\mathrm{T}}
\end{array}\right]^{\mathrm{T}}=-\mathbf{J}^{-1} \mathbf{g}^{(n+1)},
$$

where $\mathbf{g}$ denotes the residual of Eq. (21), and can be expressed by

$$
\mathbf{g}=\left[\begin{array}{c}
\mathbf{M}(t) \ddot{\mathbf{q}}_{D}+\boldsymbol{\Phi}_{, \mathbf{q}_{D}}^{\mathrm{T}} \lambda-\mathbf{Q}\left(\dot{\mathbf{q}}_{D}, \mathbf{q}_{D}, t\right) \\
\mathbf{\Phi}\left(\mathbf{q}_{D}, t\right)
\end{array}\right],
$$

and $\mathbf{J}$, the Jacobian matrix of the vector $\mathbf{g}$ with respect to $\mathbf{q}_{D}$ and $\lambda$, is written as

$$
\mathbf{J}=\frac{\partial \mathbf{g}}{\partial\left[\begin{array}{ll}
\mathbf{q}_{D}^{\mathrm{T}} & \lambda^{\mathrm{T}}
\end{array}\right]^{\mathrm{T}}}=\left[\begin{array}{cc}
\hat{\beta} \mathbf{M}+\hat{\gamma} \mathbf{C}+\mathbf{K} & \boldsymbol{\Phi}_{, \mathbf{q}_{D}}^{\mathrm{T}} \\
\boldsymbol{\Phi}_{, \mathbf{q}_{D}} & \mathbf{0}
\end{array}\right] .
$$

Here, $\mathbf{C}=-\mathbf{Q}_{, \dot{\mathbf{q}}_{D}}$ is the tangent damping matrix and $\mathbf{K}=\left(\mathbf{\Phi}_{, \mathbf{q}_{D}}^{\mathrm{T}} \boldsymbol{\lambda}\right)_{, \mathbf{q}_{D}}-\mathbf{Q}_{, \mathbf{q}_{D}}$ is the tangent stiffness matrix.

When solving the dynamic equation (21) by using the generalized- $\alpha$ method, one 
should pay special attention to the lengths of the boundary elements so as to avoid excessively long or excessively short lengths. As excessively long lengths of boundary elements can reduce the accuracy of the computation result, while the excessively short case can result in the singularity of the stiffness matrix.

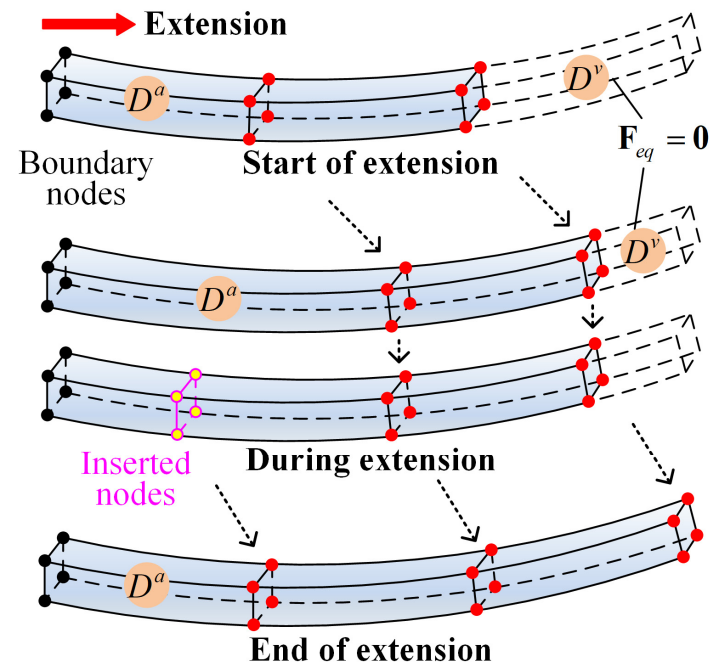

(a)

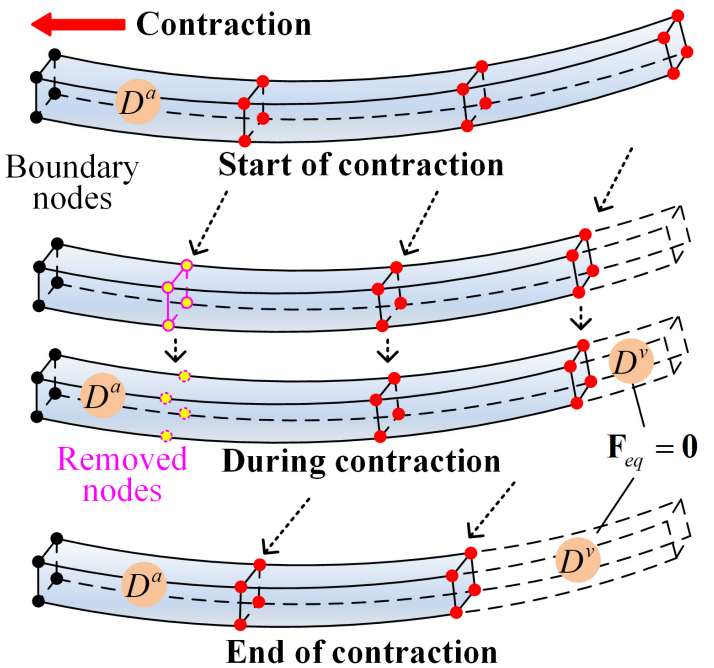

(b)

Fig. 2. Treatment of the boundary element and definition of the virtual design domain: (a) extension case; (b) contraction case.

As illustrated in Fig. 2(a), when the length of the boundary element $L_{b}$ is longer than a specified maximum length $L_{\max }$, four new nodes are inserted to divide the boundary element into two. The vectors of the generalized coordinates $\mathbf{q}_{\text {insert }}$, generalized velocities $\dot{\mathbf{q}}_{\text {insert }}$, generalized accelerations $\ddot{\mathbf{q}}_{\text {insert }}$ as well as the auxiliary vector $\mathbf{a}_{\text {insert }}$ of an inserted node can be computed as follows

$$
\begin{aligned}
\mathbf{q}_{\text {insert }} & =\left[\begin{array}{lllll}
\mathbf{q}_{e}^{\mathrm{T}} \mathbf{N}_{e}^{\mathrm{T}} & \mathbf{q}_{e}^{\mathrm{T}} \mathbf{N}_{e, x}^{\mathrm{T}} & \mathbf{q}_{e}^{\mathrm{T}} \mathbf{N}_{e, y}^{\mathrm{T}} & \mathbf{q}_{e}^{\mathrm{T}} \mathbf{N}_{e, h}^{\mathrm{T}} & h_{\text {insert }}
\end{array}\right]^{\mathrm{T}}, \\
\dot{\mathbf{q}}_{\text {insert }} & =\left[\begin{array}{lllll}
\dot{\mathbf{q}}^{\mathrm{T}} \mathbf{N}^{\mathrm{T}} & \dot{\mathbf{q}}^{\mathrm{T}} \mathbf{N}_{, x}^{\mathrm{T}} & \dot{\mathbf{q}}^{\mathrm{T}} \mathbf{N}_{, y}^{\mathrm{T}} & \dot{\mathbf{q}}^{\mathrm{T}} \mathbf{N}_{, h}^{\mathrm{T}} & \dot{h}_{\text {insert }}
\end{array}\right]^{\mathrm{T}}, \\
\ddot{\mathbf{q}}_{\text {insert }} & =\left[\begin{array}{lllll}
\ddot{\mathbf{q}}^{\mathrm{T}} \mathbf{N}^{\mathrm{T}}+\ddot{\mathbf{r}}_{h}^{\mathrm{T}} & \ddot{\mathbf{q}}^{\mathrm{T}} \mathbf{N}_{, x}^{\mathrm{T}}+\ddot{\mathbf{r}}_{h, x}^{\mathrm{T}} & \ddot{\mathbf{q}}^{\mathrm{T}} \mathbf{N}_{, y}^{\mathrm{T}}+\ddot{\mathbf{r}}_{h, y}^{\mathrm{T}} & \ddot{\mathbf{q}}^{\mathrm{T}} \mathbf{N}_{, h}^{\mathrm{T}}+\ddot{\mathbf{r}}_{h, h}^{\mathrm{T}} & \ddot{h}_{\text {insert }}
\end{array}\right]^{\mathrm{T}}, \\
\mathbf{a}_{\text {insert }} & =\left(\begin{array}{ll}
\left.1-\alpha_{f}\right) /\left(1-\alpha_{m}\right) \ddot{\mathbf{q}}_{i n s e r t} .
\end{array}\right.
\end{aligned}
$$

On the contrary, when the length of the boundary element $L b$ is shorter than a specified minimum length $L \mathrm{~min}$, the two boundary elements merge into one by simply removing the four inner nodes, as illustrated in Fig. 2(b).

When inserting or removing nodes, the deformed configuration of the flexible variable-length structure may change a little bit around the areas of the inserted or removed nodes. In future work, the error analysis will be used to improve the approach for inserting or removing nodes. Inserting or removing nodes may also changes the dimension of the system and result in the dimensional change of the ESL vectors. The actual design domain $D^{a}$ and virtual design domain $D^{v}$, which will be explained in detail in subsection 3.2, are therefore introduced to overcome this difficulty. 


\section{Optimization formulation of a variable-length structure}

In this section, the weakly coupled optimization formulation of a variable-length structure is presented. The detailed derivation of the ESL sets and a brief optimization computation flow are also given. For the fully coupled optimization formulation, the readers can refer to references [3, 16-18].

\subsection{The weakly coupled optimization formulation}

In the weakly coupled method or the ESL method [16, 19-25], the topology optimization of a variable-length structure is performed based on the static analysis. During each iteration, one only needs to solve the following static equilibrium equation for $N_{t}$ time steps

$$
\mathbf{F}_{e}\left(\mathbf{b}, \mathbf{q}_{s}\left(t_{i}\right)\right)-\mathbf{F}_{e q}\left(t_{i}\right)=\mathbf{0},\left(i=1,2, \cdots, N_{t}\right),
$$

where $t_{i}$ represents the $i$-th time step, $N_{t}$ is the total number of time steps, $\mathbf{q}_{s}$ is the vector of generalized coordinates from static analysis, $\mathbf{F}_{e}$ and $\mathbf{F}_{e q}$ denote the vectors of generalized elastic forces and the ESL, respectively. It is worth noting that the equilibrium equation in Eq. (35) of a specific variable-length structure is isolated from the FMBS as shown in Eq. (21). Boundary conditions, therefore, have to be added to avoid the singularity of the stiffness matrix $[15,20]$.

According to the ESL method, the topology optimization for dynamic response of the variable-length structure can be transferred into a static one under multiple loading cases in the following way

$$
\begin{array}{ll}
\text { find } & \mathbf{b} \in \mathbb{R}^{N_{b}} \\
\text { to minimize } & f_{E}\left(\mathbf{b}, \mathbf{q}_{S}\right) \\
\text { subject to } & \mathbf{F}_{e}\left(\mathbf{b}, \mathbf{q}_{S}\left(t_{i}\right)\right)-\mathbf{F}_{e q}\left(t_{i}\right)=\mathbf{0}, \\
& \left(i=1,2, \cdots, N_{t}\right) \\
& V(\mathbf{b})-V_{\max } \leq 0 \\
& \mathbf{b} \subset \mathcal{U}_{\mathbf{b}}
\end{array}
$$

where $\mathbf{b}=\left[\begin{array}{llll}b_{1} & b_{2} & \cdots & b_{N_{b}}\end{array}\right]^{\mathrm{T}}$ is the vector of $N_{b}$ design variables. In different methods of topology optimization, b has their meanings. For example, it may cover the densities of each finite element in the density method, the level set function values of each node in the level set method and the positions and shapes of each component in the MMC method.

In Eq. (36), for the stiffness design, the objective function $f_{E}\left(\mathbf{b}, \mathbf{q}_{S}\right)$ is defined as the weighted summation of the compliances of the variable-length structure at all time steps as follows

$$
f_{E}\left(\mathbf{b}, \mathbf{q}_{S}\right)=\sum_{i=1}^{N_{t}} \int_{V_{0}} \omega_{i} \boldsymbol{\sigma}_{i}\left(\mathbf{b}, \mathbf{q}_{S}\right)^{\mathrm{T}} \boldsymbol{\varepsilon}_{i}\left(\mathbf{b}, \mathbf{q}_{S}\right) \mathrm{d} V
$$


where $\omega_{i}$ is the weight coefficient for the $i$-th time step and can be determined via the expert evaluation method of weights [19]. The effect of the weights on the optimization results, however, is not the focus of this study. Hence, they are chosen to be the same for all the time steps in this study. $V_{0}$ is the initial undeformed volume of the variablelength structure. $\boldsymbol{\sigma}$ is the Piola-Kirchhoff stress tensor of the second kind and $\boldsymbol{\varepsilon}$ is the Green-Lagrange strain tensor. Minimizing the compliance $f_{E}$ is equivalent to maximizing the stiffness of the structure.

Among the constraints in Eq. (36), the first one is the equivalent static analysis of the variable-length structure. $V(\mathbf{b})-V_{\max } \leq 0$ is the inequality volume constraint, which indicates that the volume ratio $V$ of the variable-length structure should be no more than the specified volume fraction $V_{\max } . \mathcal{U}_{\mathbf{b}}$ in Eq. (36) is the design space that b belongs to.

Compared with the fully coupled optimization method, the ESL method turns out to be more efficient in computation for the cases in [18, 19], and it has been successfully applied to many dynamic response optimization problems [3, 15, 17-20, 23, 24, 49, 50]. The ESL method is therefore employed in this study and reevaluated in subsection 3.2 to deal with the variable-length characteristics.

\subsection{Derivation of the ESL sets}

For the optimization of a constant-length flexible structure in an FMBS, the ESL sets have been well-defined as the static loads that generate the same deformations of the structure from the static analysis as those from the nonlinear dynamic analysis based on ANCF $[15,19]$ at any time steps as follows

$$
\mathbf{F}_{e q}\left(t_{i}\right)=\mathbf{K}\left(\mathbf{b}, \mathbf{q}_{D}\left(t_{i}\right)\right)\left(\mathbf{q}_{D}\left(t_{i}\right)-\mathbf{q}_{R}\left(t_{i}\right)\right),\left(i=1,2, \cdots, N_{t}\right) .
$$

Here, $t_{i}$ denotes the $i$-th time step and $N_{t}$ is the total number of time steps. $\mathbf{q}_{D}$ and $\mathbf{q}_{R}$ represent the vectors of generalized coordinates from the dynamic analysis of FMBS and the rigid multibody dynamic analysis, respectively. $\mathbf{K}$ is the nonlinear stiffness matrix of ANCF, which is a function of the vector of generalized coordinates $\mathbf{q}_{D}$ and the vector of design variables $\mathbf{b}$.

Motivated by the original definition of the ESL sets in Eq. (38), the ESL sets for a variable-length structure are redefined in this study. They are defined as the static loads that generate the same deformations of the active structure from the static analysis as those from the nonlinear dynamic analysis based on ALE-ANCF at any time steps in the actual design domain, and are always zeros in the virtual design domain. As shown in Fig. 2, the actual design domain $D^{a}$ is the stretching domain for the extension case, and the uncontracted domain for the contraction case of the variable-length structure. The virtual design domain $D^{v}$ is the unstretched domain for the extension case and the contracting domain for the contraction case. The aforementioned active structure, hence, is corresponding to the actual design domain $D^{a}$ at a specific time step. The whole design domain $D=D^{a} \cup D^{v}$, representing the whole variable-length structure to be optimized, is constant in length. 
According to the definition of the ESL sets for a variable-length structure, the ESL sets can be separately computed in the two domains as

$$
\begin{aligned}
& \mathbf{F}_{e q}\left(\mathbf{r}, t_{i}\right)=\mathbf{K}^{a}\left(\mathbf{b}, \mathbf{q}_{D}^{a}\left(t_{i}\right)\right)\left(\mathbf{q}_{D}^{a}\left(t_{i}\right)-\mathbf{q}_{R}^{a}\left(t_{i}\right)\right),\left(\mathbf{r} \in D^{a}\right), \\
& \mathbf{F}_{e q}\left(\mathbf{r}, t_{i}\right)=\mathbf{0},\left(\mathbf{r} \in D^{v}\right),
\end{aligned}
$$

where $i=1,2, \cdots, N_{t}, \mathbf{q}_{D}^{a}$ and $\mathbf{q}_{R}^{a}$ are the vectors of generalized coordinates in the actual design domain $D^{a} . \mathbf{r}$ is an arbitrary point in the whole design domain $D . \mathbf{K}^{a}$ is the tangent stiffness matrix in $D^{a}$, and according to Eq. (30) can be expressed as

$$
\mathbf{K}^{a}=\left(\mathbf{\Phi}_{, \mathbf{q}_{D}^{a}}^{\mathrm{T}} \lambda\right)_{, \mathbf{q}_{D}^{a}}-\mathbf{Q}_{, \mathbf{q}_{D}^{a}} \text {. }
$$

For the deployment simulation of a variable-length structure undergoing large overall motion, only linear kinematic constraints are considered. Therefore, the secondorder partial derivatives of $\boldsymbol{\Phi}$ with respect to $\mathbf{q}_{D}^{a}$ will vanish, that is, $\left(\boldsymbol{\Phi}_{, \mathbf{q}_{D}^{a}}^{\mathrm{T}} \boldsymbol{\lambda}\right)_{, \mathbf{q}_{D}^{a}}=\mathbf{0}$. According to Eq. (22), $\mathbf{Q}_{, \mathbf{q}_{D}^{a}}$ can be expressed as

$$
\mathbf{Q}_{, \mathbf{q}_{D}^{a}}=\mathbf{F}_{f, \mathbf{q}_{D}^{a}}-\mathbf{F}_{a, \mathbf{q}_{D}^{a}}-\mathbf{F}_{e, \mathbf{q}_{D}^{a}} .
$$

At a specific time step, the length of the structure is constant, i.e., $\dot{h}_{1}=0$ and $\dot{h}_{2}=0$. $\mathbf{F}_{a}=\mathbf{0}$ can then be obtained according to Eqs. (18) and (9). In general, the vector of generalized external forces $\mathbf{F}_{f}$ is neglected. Therefore, Eq. (41) can be rewritten as

$$
\mathbf{K}^{a}=\mathbf{F}_{e, \mathbf{q}_{D}^{a}}
$$

which indicates that in the computation of the ESL sets for a variable-length structure, the tangent stiffness matrix in Eq. (39) is identical to the Jacobian of the vector of system generalized elastic forces.

\subsection{The ESL-based optimization computation flow}

In this section, the overall algorithm steps for the optimization of a $3 \mathrm{D}$ variablelength structure by using the ESL method are briefly listed as follows:

Step 1: Initialize the optimization cycle number $K=0$, and the vector of design variables $\mathbf{b}^{(K)}=\mathbf{b}^{(0)}$.

Step 2: Establish the FMBS model (21) for a 3D variable-length structure via the ALE-ANCF.

Step 3: Integrate the dynamic equation (21) of the 3D FMBS via the generalized$\alpha$ method.

Step 4: Perform the ESL analysis based on the dynamic responses of Step 3.

Step 5: Solve the optimization problem (36) of static response via the Method of Moving Asymptotes (MMA) [51].

Step 6: Update the vector of design variables $\mathbf{b}^{(K-1)}=\mathbf{b}^{(K)}, \mathbf{b}^{(K)}=\mathbf{b}^{(K+1)}$, and cycle number $K=K+1$.

Step 7: Estimate the convergence criterion $\left\|\mathbf{b}^{(K)}-\mathbf{b}^{(K-1)}\right\| \leq \varepsilon(K \geq 1)$, where the 
convergence parameter $\varepsilon$ is a small positive number. If the change of the design variables is not greater than $\varepsilon$, end the optimization process. Otherwise, go to Step 2.

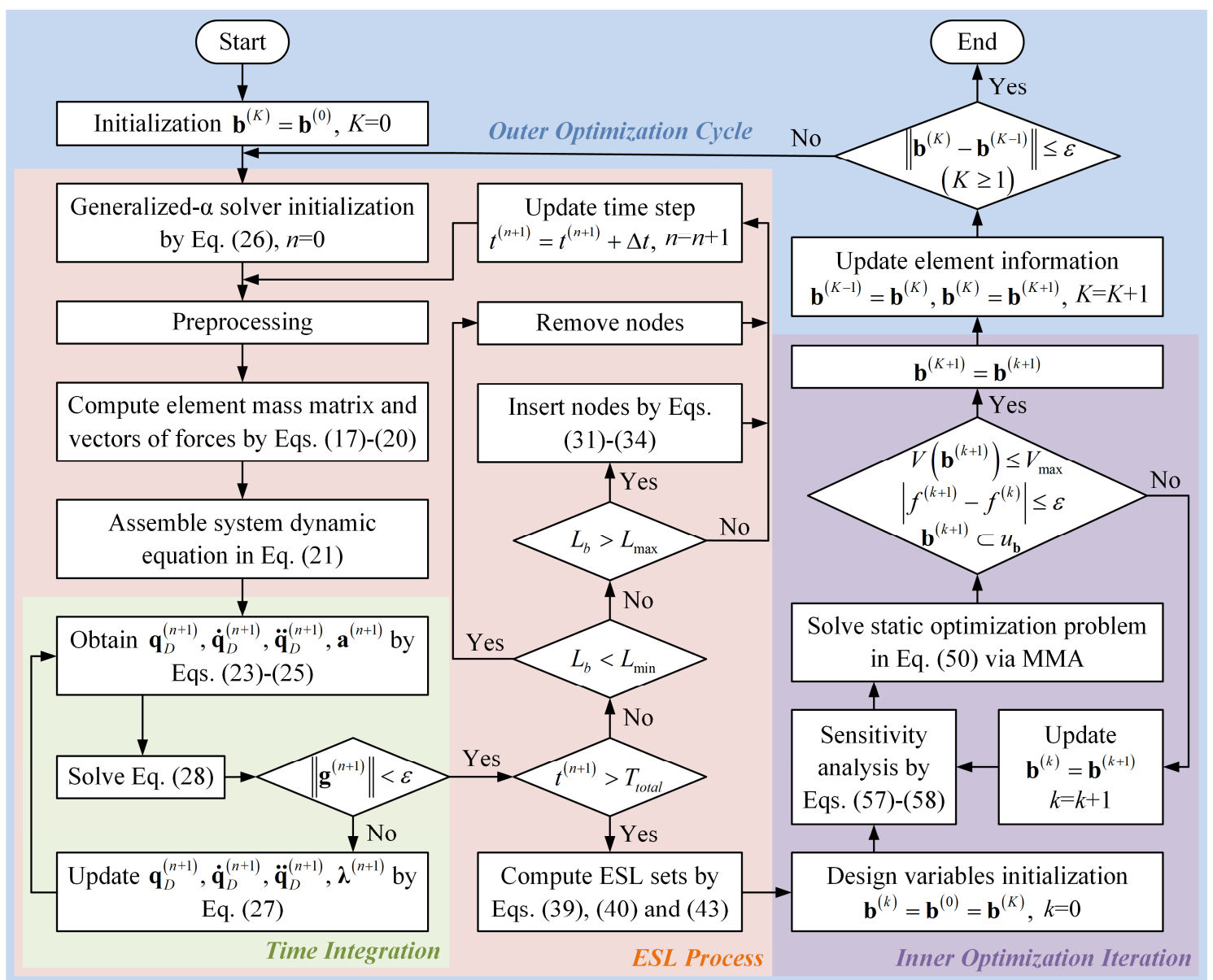

Fig. 3. The flowchart of the ESL-based computation for the optimization of a variable-length structure.

Among all the steps, Step 1 is called the initialization. Step 2, Step 3 and Step 4 are called the ESL process. Step 5 is called the inner optimization iteration and will be addressed in Section 4, where a new simultaneous topology and size optimization approach is proposed. The process from Step 7 to Step 2 is called the outer optimization cycle $[19,20]$. The detailed optimization computation flow for a $3 \mathrm{D}$ variable-length structure by using the ESL method is illustrated in Fig. 3.

\section{Simultaneous topology and size optimization via MMC}

In this section, a simultaneous topology and size optimization approach is proposed to solve the optimization problem (36) of static response based on the MMC framework. The main idea of the optimization approach is realized by linking some specific design variables and modifying their sensitivities to be identical during the optimization iteration. According to this approach, a 3D variable-length structure can be designed as a homogeneous or a heterogeneous periodic structure depending on the number of linked design variables. 


\subsection{The basic concept of MMC}

For 3D optimization problems, 3D structural components are used as the basic building blocks. In this study, a cuboid component is employed, as shown in Fig. 4(a). For the $j$-th component, the topology description function can be mathematically expressed as [39]

$$
\chi_{j}(X, Y, Z)=1-\left(\frac{X}{L_{1}}\right)^{p}-\left(\frac{y}{L_{2}}\right)^{p}-\left(\frac{Z}{L_{3}}\right)^{p},
$$

where $j=1,2, \cdots, N_{c}$ and $N_{c}$ is the number of the structural components. $p$ is a large positive even integer and here $p=6 . L_{1}, L_{2}$, and $L_{3}$ are the semilength, semiwidth, and semiheight of the cuboid component. $O-X Y Z$ and $o-x y z$ represent the global frame of coordinates and local frame of coordinates, respectively. The local coordinates $(x, y, z)$ and the global coordinates $(X, Y, Z)$ have the following relationship

$$
\left[\begin{array}{l}
x \\
y \\
z
\end{array}\right]=\mathbf{R}\left[\begin{array}{c}
X-X_{0} \\
Y-Y_{0} \\
Z-Z_{0}
\end{array}\right]
$$

with $\left(X_{0}, Y_{0}, Z_{0}\right)$ denoting the global coordinates of the component centroid.

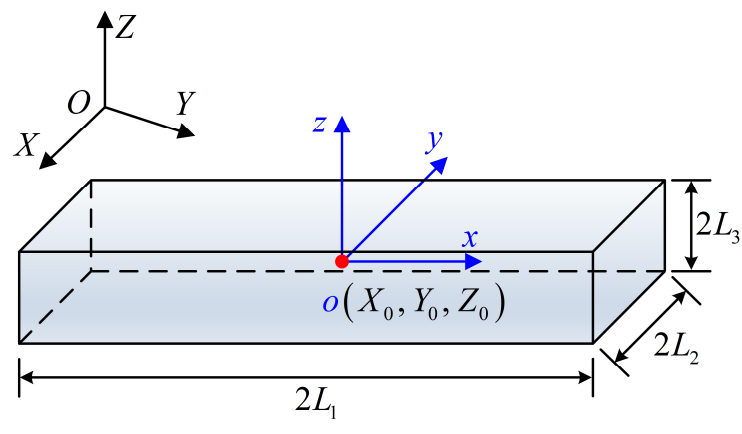

(a)

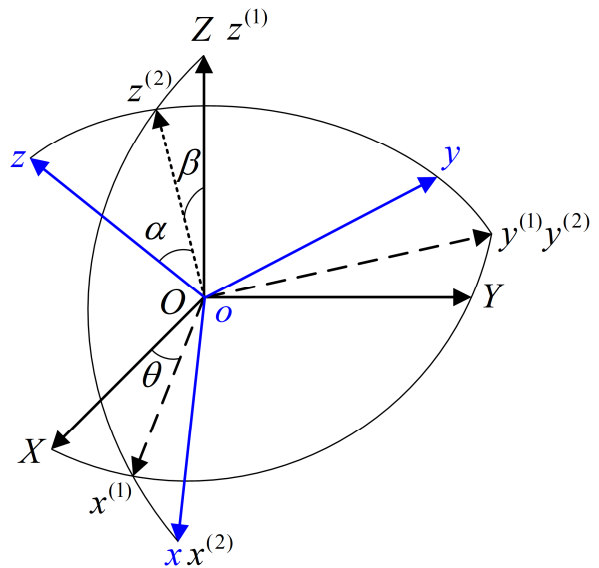

(b)

Fig. 4. Definition of the design variables for a cuboid component: (a) positions and shapes; (b) orientations.

In Eq. (45), $\mathbf{R}$ is the rotation transformation matrix and computed by

$$
\mathbf{R}=\left[\begin{array}{ccc}
\cos \beta \cos \theta & -\cos \beta \sin \theta & \sin \beta \\
\sin \alpha \sin \beta \cos \theta+\cos \alpha \sin \theta & -\sin \alpha \sin \beta \sin \theta+\cos \alpha \cos \theta & -\sin \alpha \cos \beta \\
-\cos \alpha \sin \beta \cos \theta+\sin \alpha \sin \theta & \cos \alpha \sin \beta \sin \theta+\sin \alpha \cos \theta & \cos \alpha \cos \beta
\end{array}\right]
$$

where $\alpha, \beta$ and $\theta$ are a kind of Tait-Bryant angles. As illustrated in Fig. 4(b), the global coordinate system $O-X Y Z$ rotates about $Z$-axis, $y^{(1)}$-axis and $x^{(2)}$-axis by angles of $\theta, \beta$ and $\alpha$, respectively to obtain the local coordinate system $o-x y z$ assuming that $O$ and $o$ coincide with each other.

According to Eqs. (44)-(46), the vector of design variables for the $j$-th component can be defined as 


$$
\mathbf{b}_{j}=\left[\begin{array}{lllllllll}
X_{0} & Y_{0} & Z_{0} & L_{1} & L_{2} & L_{3} & \sin \alpha & \sin \beta & \sin \theta
\end{array}\right]^{\mathrm{T}} .
$$

Once $\mathbf{b}_{j}$ is obtained, the position, shape, and orientation of the $j$-th component can be determined. The material domain $\Omega_{j}$ occupied by the $j$-th component, the material interface $\partial \Omega_{j}$ of the $j$-th component, and the void domain $D \backslash \Omega_{j}$ corresponding to the $j$-th component can then be described according to Eq. (44) as

$$
\left\{\begin{array}{l}
\chi_{j}(\mathbf{r})>0 \Leftrightarrow \mathbf{r} \in \Omega_{j} \\
\chi_{j}(\mathbf{r})=0 \Leftrightarrow \mathbf{r} \in \partial \Omega_{j} \\
\chi_{j}(\mathbf{r})<0 \Leftrightarrow \mathbf{r} \in D \backslash \Omega_{j}
\end{array}\right.
$$

where $\mathbf{r}$ is an arbitrary point in the design domain $D \in \mathbb{R}^{3}$, and $\chi_{j}$ is the topology description function for the $j$-th component defined in Eq. (44). For the entire variablelength structure, the topology description function $\chi$ and the material domain $\Omega$ can then be obtained by

$$
\chi=\max \left(\chi_{1}, \chi_{2}, \cdots, \chi_{N_{c}}\right), \Omega=\bigcup_{j=1}^{N_{c}} \Omega_{j} .
$$

\subsection{The periodic structure optimization formulation}

For a 3D deployable variable-length structure, periodic structure design is a promising choice as the periodic structure has many merits, such as high manufacturing and assembling efficiency, high deployment repeatability and retraction capability and so on. According to the feature sizes of the lattice cells, the periodic structures can be divided into homogeneous periodic structures and heterogeneous periodic structures [5]. For a homogeneous periodic structure, all the lattice cells have exactly the same topology and feature size, while for a heterogeneous periodic structure, they only have the same topology but different feature sizes like the thicknesses.

Based on the MMC framework, the periodic structure design for a 3D variablelength structure can be easily implemented. Firstly, the number of the lattice cells is determined by the designers. Secondly, the initial configurations of the structural components are set the same for all the lattice cells. Thirdly, some of the design variables in different lattice cells are linked and the sensitivities with respect to the linked design variables are modified to be identical. The optimization formulation in Eq. (36) can now be rewritten as follows

$$
\begin{array}{ll}
\text { find } & \mathbf{b} \in \mathbb{R}^{N_{b}} \\
\text { to minimize } & f_{E}\left(\mathbf{b}, \mathbf{q}_{S}\right) \\
\text { subject to } & \mathbf{K}\left(\mathbf{b}, \mathbf{q}_{D}\left(t_{i}\right)\right)\left(\mathbf{q}_{s}\left(t_{i}\right)-\mathbf{q}_{R}\left(t_{i}\right)\right)-\mathbf{F}_{e q}\left(t_{i}\right)=\mathbf{0} \quad\left(i=1,2, \cdots, N_{t}\right) \\
& \frac{\partial \hat{f}}{\partial\left(\mathbf{b}_{j_{s}}\right)_{k}}=\frac{1}{N_{l}} \sum_{s=1}^{N_{l}} \frac{\partial f}{\partial\left(\mathbf{b}_{j_{s}}\right)_{k}}\left(k \in[1,9], s=1,2, \cdots, N_{l}\right) \\
& V(\mathbf{b})-V_{\max } \leq 0 \\
& \mathbf{b} \subset \mathcal{U}_{\mathbf{b}}
\end{array}
$$


where $\quad \mathbf{b}=\left[\begin{array}{llll}\mathbf{b}_{1}^{\mathrm{T}} & \mathbf{b}_{2}^{\mathrm{T}} & \cdots & \mathbf{b}_{N_{c}}^{\mathrm{T}}\end{array}\right]^{\mathrm{T}}$ is the vector of design variables with $\mathbf{b}_{j}$ $\left(j=1,2, \cdots, N_{c}\right)$ denoting the vector of design variables of the $j$-th component in Eq. (47). $N_{c}$ is the number of components and the total number of design variables $N_{b}=9 N_{c}$. $\left(\mathbf{b}_{j}\right)_{k}(k=1,2, \cdots, 9)$ represents the $k$-th design variable of the $j$-th component. $N_{t}$ is the number of time steps and $N_{l}$ is the number of lattice cells.

In Eq. (50), $\frac{\partial \hat{f}}{\partial\left(\mathbf{b}_{j_{s}}\right)_{k}}=\frac{1}{N_{l}} \sum_{s=1}^{N_{l}} \frac{\partial f}{\partial\left(\mathbf{b}_{j_{s}}\right)_{k}}$ is an additional constraint that modifies the sensitivities with respect to some of the design variables in different lattice cells. Here, $f$ is the objective function $f_{E}\left(\mathbf{b}, \mathbf{q}_{S}\right)$ or the volume constraint $V(\mathbf{b})$. $\partial f / \partial\left(\mathbf{b}_{j_{s}}\right)_{k}$ is the sensitivity with respect to the $k$-th design variable of the $j_{s}$-th component, while $\partial \hat{f} / \partial\left(\mathbf{b}_{j_{s}}\right)_{k}$ is the corresponding modified sensitivity. It can be seen that the modified sensitivities with respect to the $k$-th design variables of the $j_{1}$-th , $j_{2}$-th $, \cdots, j_{N_{l}}$-th $\left(1 \leq j_{1}<j_{2}<\cdots<j_{N_{l}} \leq N_{c}\right)$ components are identical and equal to the average value of their original sensitivities. Hence, we say that these design variables are linked.

It is worth mentioning that "link" does not mean that $\left(\mathbf{b}_{j_{1}}\right)_{k}=\left(\mathbf{b}_{j_{2}}\right)_{k}=\cdots=\left(\mathbf{b}_{j_{N_{l}}}\right)_{k}$, but that these linked design variables will have the same sensitivity during optimization iteration. For example, if $k=1$ or 2 or 3 , the linked design variables are the positions of the components in different lattice cells and obviously, they will have different values. It is also noteworthy that a component can be either fully or partly linked to other components owing to the explicit topology description of MMC. On one hand, if $k=1,2,3,4,5,6,7,8,9$ in Eq. (50), all the nine design variables of a component are linked to other components in different lattice cells so that a homogeneous periodic structure can be obtained. On the other hand, if $k=1,2,3,4,7,8,9$ in Eq. (50), only the design variables for the positions, semilength and orientations of a component are linked to other components. The semiwidth and semiheight of the components in different lattice cells can be freely optimized. In this way, a heterogeneous periodic structure can be obtained after optimization.

\subsection{Sensitivity analysis}

In the MMC method, the objective function $f_{E}\left(\mathbf{b}, \mathbf{q}_{S}\right)$ in Eq. (37) and volume ratio $V(\mathbf{b})$ can be respectively computed by

$$
\begin{aligned}
& f_{E}\left(\mathbf{b}, \mathbf{q}_{S}\right)=\sum_{i=1}^{N_{t}} \int_{V_{0}} \omega_{i} H(\chi) \boldsymbol{\sigma}_{i}\left(\mathbf{b}, \mathbf{q}_{S}\right)^{\mathrm{T}} \boldsymbol{\varepsilon}_{i}\left(\mathbf{b}, \mathbf{q}_{S}\right) \mathrm{d} V, \\
& V(\mathbf{b})=\frac{1}{V_{0}} \int_{V_{0}} H(\chi) \mathrm{d} V,
\end{aligned}
$$


where $H(\chi)$ is the Heaviside function, and its derivative function is the Dirac delta function $\delta(\chi)$ defined by

$$
\delta(\chi)=\frac{\mathrm{d} H}{\mathrm{~d} \chi}, H(\chi)=\left\{\begin{array}{ll}
1, & (\chi \geq 0) \\
0, & (\chi<0)
\end{array} .\right.
$$

According to the work by Zhang et al. [39], the derivatives of the objective function (51) and volume ratio (52) with respect to the design variables $\left(\mathbf{b}_{j}\right)_{k}$ can be expressed as

$$
\begin{aligned}
& \frac{\partial f_{E}}{\partial\left(\mathbf{b}_{j}\right)_{k}}=-\sum_{i=1}^{N_{t}} \int_{V_{0}} \omega_{i} \delta_{j}(\chi) \boldsymbol{\sigma}_{i}\left(\mathbf{b}, \mathbf{q}_{S}\right)^{\mathrm{T}} \boldsymbol{\varepsilon}_{i}\left(\mathbf{b}, \mathbf{q}_{S}\right) \frac{\partial \chi_{j}}{\partial\left(\mathbf{b}_{j}\right)_{k}} \mathrm{~d} V, \\
& \frac{\partial V}{\partial\left(\mathbf{b}_{j}\right)_{k}}=\frac{1}{V_{0}} \int_{V_{0}} \delta_{j}(\chi) \frac{\partial \chi_{j}}{\partial\left(\mathbf{b}_{j}\right)_{k}} \mathrm{~d} V,
\end{aligned}
$$

where $j=1,2, \cdots, N_{c}$ and $k=1,2, \cdots, 9 . \delta_{j}(\chi)$ is defined as

$$
\delta_{j}(\chi)=\min \left(\delta\left(\chi_{j}\right), \delta(\chi)\right),\left(j=1,2, \cdots, N_{c}\right) .
$$

As the topology description function $\chi_{j}$ in Eq. (44) can be explicitly described by the vector of design variables $\mathbf{b}_{j}$ according to Eqs. (44)-(47), the formulae of $\partial \chi_{j} / \partial\left(\mathbf{b}_{j}\right)_{k}$ in Eqs. (54) and (55) can be easily obtained, which will not be presented in this study.

If the $k$-th design variables $(k \in[1,9])$ of $j_{1}$-th,$j_{2}$-th $, \cdots, j_{N_{l}}$-th $\left(1 \leq j_{1}<j_{2}<\cdots<j_{N_{l}} \leq N_{c}\right)$ components are linked, the modified sensitivities of the objective function $f_{E}\left(\mathbf{b}, \mathbf{q}_{S}\right)$ and volume ratio $V(\mathbf{b})$ with respect to those linked design variables can be computed in the following way

$$
\begin{aligned}
\frac{\partial \hat{f}_{E}}{\partial\left(\mathbf{b}_{j_{1}}\right)_{k}} & =\frac{\partial \hat{f}_{E}}{\partial\left(\mathbf{b}_{j_{2}}\right)_{k}}=\cdots=\frac{\partial \hat{f}_{E}}{\partial\left(\mathbf{b}_{j_{N_{l}}}\right)_{k}} \\
& =-\frac{1}{N_{l}} \sum_{s=1}^{N_{l}} \sum_{i=1}^{N_{t}} \int_{V_{0}} \omega_{i} \delta_{j_{s}}(\chi) \boldsymbol{\sigma}_{i}\left(\mathbf{b}, \mathbf{q}_{s}\right)^{\mathrm{T}} \boldsymbol{\varepsilon}_{i}\left(\mathbf{b}, \mathbf{q}_{s}\right) \frac{\partial \chi_{j_{s}}}{\partial\left(\mathbf{b}_{j_{s}}\right)_{k}} \mathrm{~d} V, \\
\frac{\partial \hat{V}}{\partial\left(\mathbf{b}_{j_{1}}\right)_{k}} & =\frac{\partial \hat{V}}{\partial\left(\mathbf{b}_{j_{2}}\right)_{k}}=\cdots=\frac{\partial \hat{V}}{\partial\left(\mathbf{b}_{j_{N_{l}}}\right)_{k}}=\frac{1}{N_{l} V_{0}} \sum_{s=1}^{N_{l}} \int_{V_{0}} \delta_{j_{s}}(\chi) \frac{\partial \chi_{j_{s}}}{\partial\left(\mathbf{b}_{j_{s}}\right)_{k}} \mathrm{~d} V .
\end{aligned}
$$

\section{Numerical examples}

In this section, three numerical examples are presented to validate the proposed optimization approach for a 3D variable-length structure. The first two examples are the optimization of an axially moving structure carrying a payload. The third example is the heterogeneous periodic structure design for the deployable structures for 
supporting a practical spinning solar sail $[3,52]$.

\subsection{Non-periodic and periodic structure design for an axially moving structure}

This example is used to demonstrate the effectiveness of the simultaneous topology and size optimization approach by the non-periodic and periodic structure design for an axially moving structure. As shown in Fig. 5, the flexible axially moving structure extends from a rigid base and carries a rigid payload. The flexible structure is connected to the rigid base through a sliding joint and to the payload via four spherical joints at the four endpoints. The rigid base has a geometrical size of $0.2 \mathrm{~m} \times 0.2 \mathrm{~m} \times 0.18$ $\mathrm{m}$. The rigid payload has a mass of $1 \mathrm{~kg}$ and a geometrical size of $0.15 \mathrm{~m} \times 0.15 \mathrm{~m} \times 0.12$ $\mathrm{m}$. The flexible axially moving structure has an initial length of $0.1 \mathrm{~m}$ and a square cross-section of $0.1 \mathrm{~m} \times 0.1 \mathrm{~m}$. The extension velocity of the structure is $0.4 \mathrm{~m} / \mathrm{s}$ and the extension time is $2 \mathrm{~s}$ so that the final length of the flexible axially moving structure is $0.9 \mathrm{~m}$. The mass density of the structure material is $2700 \mathrm{~kg} / \mathrm{m}^{3}$, and Young's modulus and Poison's ratio are $5 \times 10^{8} \mathrm{~Pa}$ and 0.3 , respectively. The gravity is neglected in this example.

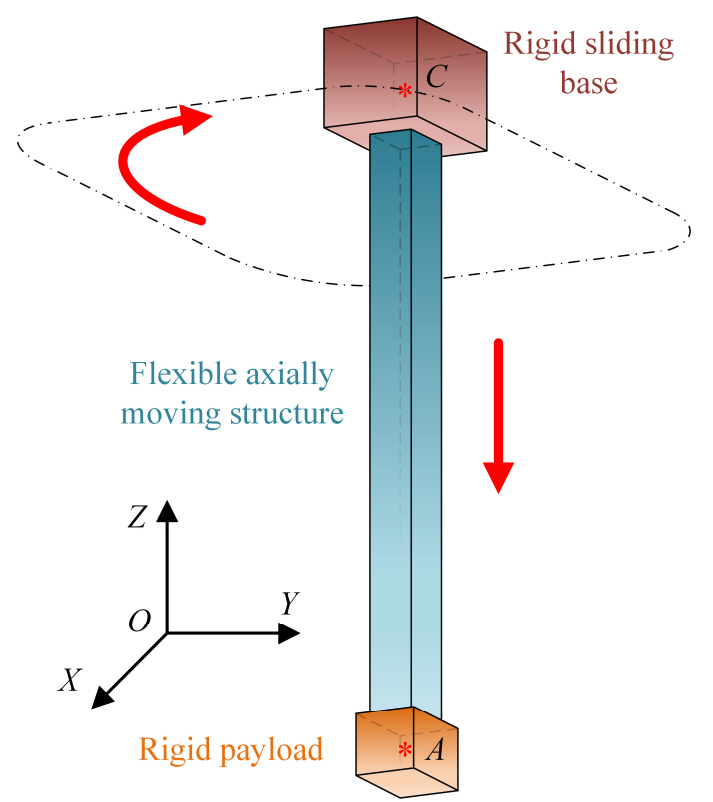

Fig. 5. An axially moving structure carrying a payload.

During the extension of the flexible structure, the rigid base moves along the dotdashed curve in Fig. 5, the equation of which is

$$
\left\{\begin{array}{l}
\left(X_{C} / 0.3\right)^{4}+\left(Y_{C} / 0.3\right)^{4}=1 \\
Z_{C}=0.09
\end{array} .\right.
$$

Here, $\left(X_{C}, Y_{C}, Z_{C}\right)$ is the coordinates of the rigid base centroid $C$, the trajectory of which is illustrated in Fig. 6(a). The speed of the rigid base is shown in Fig. 6(b) so that the base can complete a full round of the curve (59) in $2 \mathrm{~s}$.

Fig. 7 presents the configurations of the axially moving structure at four different moments during the deployment. For visualization purpose, each solid element of 
ALE-ANCF is plotted via a $4 \times 4 \times 4$ mesh size. From Fig. 7 , it can be easily seen that the number of the solid elements of ALE-ANCF used varies over time, so does the length of the boundary element. For this example, the maximum length of the boundary element $L \max =0.1 \mathrm{~m}$, which implies that in Fig. 2(a), when the length of the boundary element gets to $0.1 \mathrm{~m}$, four nodes of ALE-ANCF are inserted to equally divide the element into two elements. Fig. 8 shows the deflections of the payload centroid $A$ in both $X$ - and $Y$-directions and also gives the steady-state responses after the deployment process, i.e., $t>2 \mathrm{~s}$.

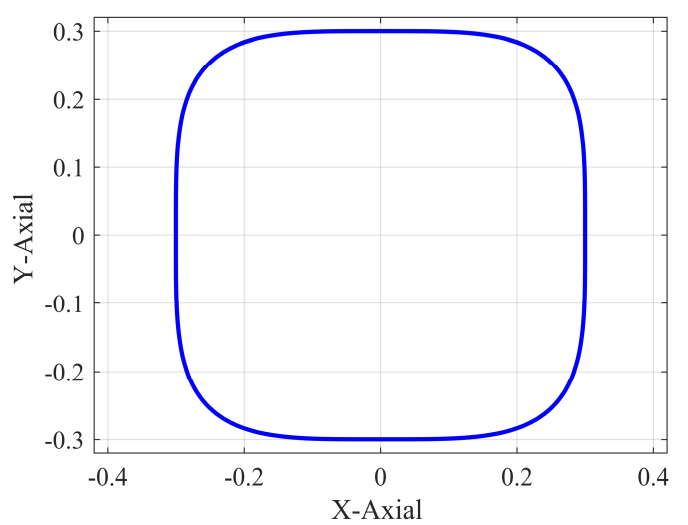

(a)

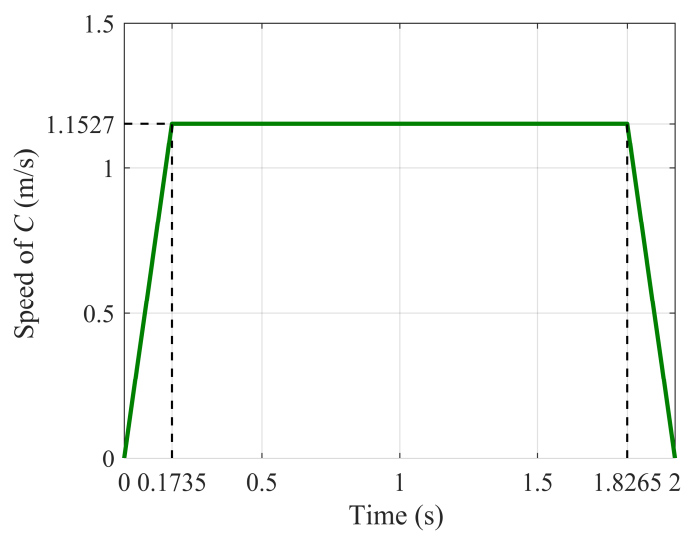

(b)

Fig. 6. Trajectory and speed of the rigid base centroid $C$ : (a) trajectory of $C$; (b) speed of $C$.



Fig. 7. Configurations of the axially moving structure at four moments.

To optimize the flexible axially moving structure during the deployment $(t=0 \sim 2$ $\mathrm{s})$, the time step size is set as $0.05 \mathrm{~s}$ for ESL analysis. Hence, there are 40 time steps altogether. As stated in subsection 3.1, the weights $\omega_{i}(i=1,2, \cdots, 40)$ are chosen to be the same for all the time steps neglecting the subjective influence of the determination of the weights. The volume fraction $V_{\max }$ is set as 0.3 for this case. In this example, three kinds of structures are designed for the axially moving structure by using the 
simultaneous topology and size optimization approach, i.e., non-periodic structure, heterogeneous periodic structure and homogeneous periodic structure. For the nonperiodic structure design, we simply neglect the additional constraint “ $\frac{\partial \hat{f}}{\partial\left(\mathbf{b}_{j_{s}}\right)_{k}}=\frac{1}{N_{l}} \sum_{s=1}^{N_{l}} \frac{\partial f}{\partial\left(\mathbf{b}_{j_{s}}\right)_{k}}, k \in[1,9]$ " in Eq. (50). For the heterogeneous and homogeneous periodic structure design, the additional constraint is considered, and $k=1,2,3,4,7,8,9$ and $k=1,2,3,4,5,6,7,8,9$, respectively.

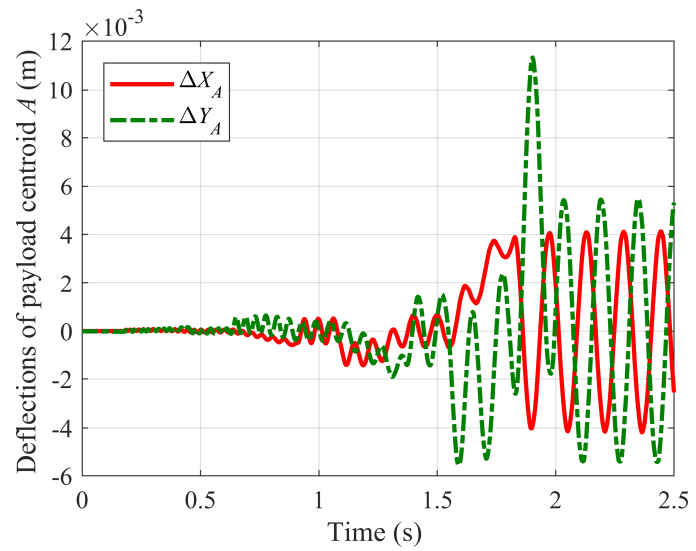

Fig. 8. Deflections of payload centroid $A$ in $X$ - and $Y$-directions.

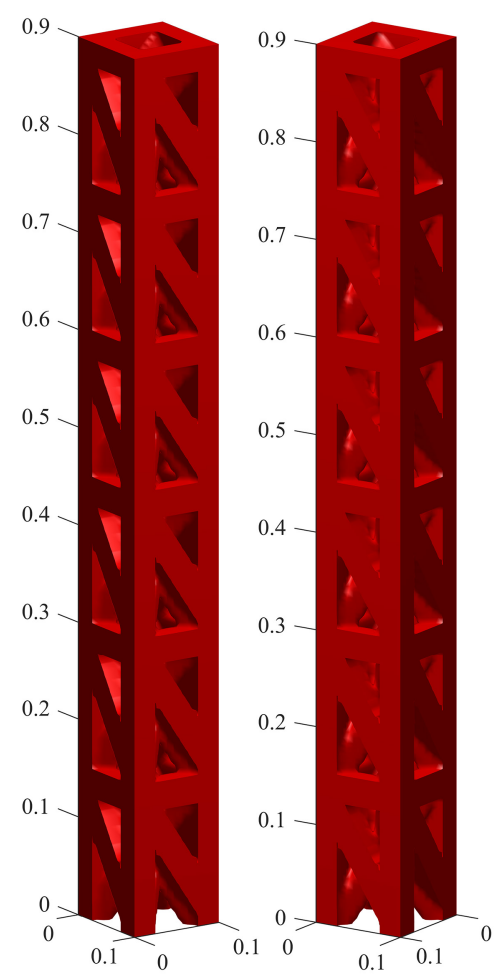

Fig. 9. Initial configuration of the components for the axially moving structure to be optimized.

Fig. 9 illustrates the initial configuration of the components for the axially moving structure, where six lattice cells are considered. In this study, the influence of the initial configurations on the optimization results is not considered, but it was considered in a previous study [23]. For visualization purpose, the initial configuration in Fig. 9 is 
presented from two different angles. In one lattice cell, 16 components are symmetrically placed and hence, each lattice cell has 144 design variables. The total number of the design variables is 864 for this example. Fig. 10 shows the optimized results of the axially moving structure. From Fig. 10, it can be obviously seen that the three optimized results have totally different topologies. The results of the non-periodic structure design in Fig. 10(a) and the heterogeneous periodic structure design in Fig. 10(b) both have more material on the top of the axially moving structure. Besides, they are mixed plate-like and truss-like structures as some of the optimized components on the top of the axially moving structure look like plates while others on the bottom look like trusses. The result of the homogeneous periodic structure design in Fig. 10(c), however, is just a kind of truss-like structure with four axially thick and strong components on the edges. According to Fig. 10(b) and (c), two kinds of periodic structures are successfully obtained with six lattice cells, which clearly validates the effectiveness of the proposed simultaneous topology and size optimization approach.

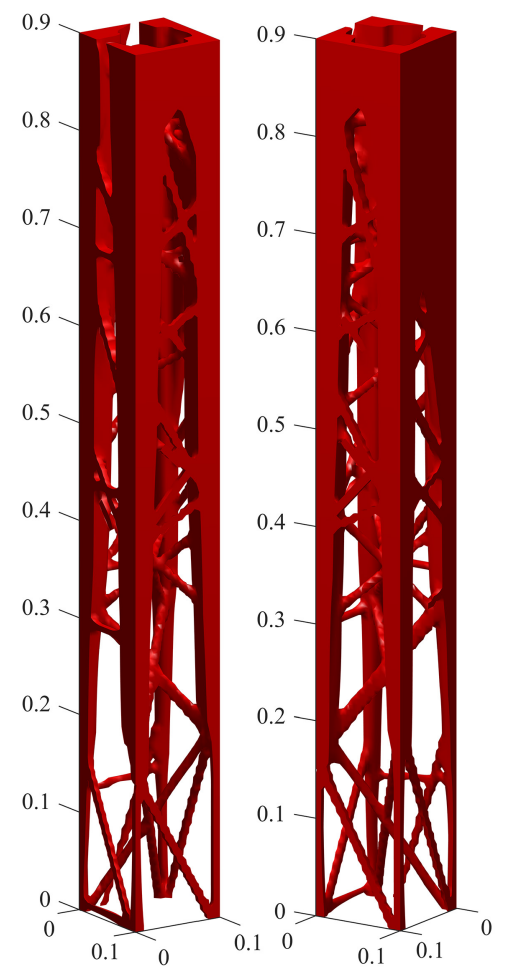

(a)

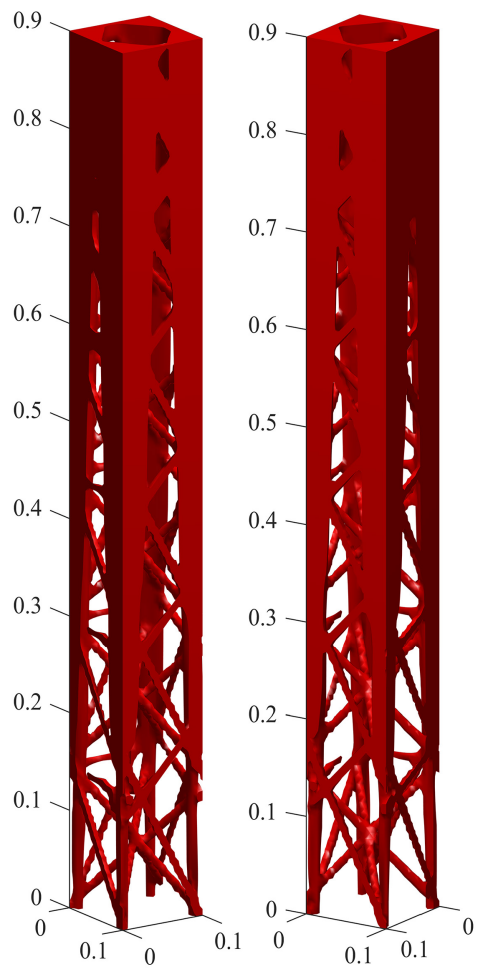

(b)

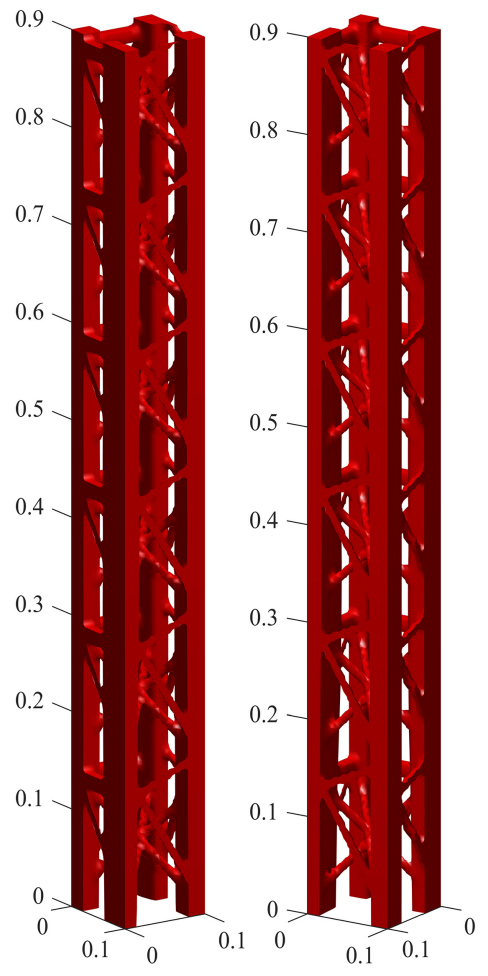

(c)

Fig. 10. Optimized results of the axially moving structure: (a) non-periodic structure design; (b) heterogeneous periodic structure design; (c) homogeneous periodic structure design.

Theoretically, the optimized result in Fig. 10(a) should be better than that in Fig. 10(b) which should be better than that in Fig. 10(c) as well. This is due to the fact that the optimization formulation for Fig. 10(a) has no constraints on the linked design variables, while the optimization formulation for Fig. 10(b) has fewer constraints on the linked design variables than that for Fig. 10(c). As additional constraints, the linked design variables will surely reduce the design feasible region. This phenomenon can also be observed in Fig. 11 which shows the iteration histories of the objective function and volume ratio for the three cases in Fig. 10. The convergent objective function value 
in Fig. 11(a) is the smallest one while the value in Fig. 11(c) is the largest. Moreover, the objective function value in Fig. 11(c) is much larger than the other two values in Fig. 11(a) and (b), in which the convergent curves are quite close to each other. This phenomenon indicates that the homogeneous periodic structure design is actually the last choice for the design of a 3D variable-length structure. Also, Fig. 11 shows that the volume constraints $(V \leq 0.3)$ are all satisfied for the three cases.

From Fig. 11, it can be found that a rather large number of iterations are needed to achieve convergence. This is actually one of the inherent properties of the MMC-based topology optimization method [37-40] as a new topology is obtained through the overlapping of the components. And the overlapping is implemented by changing the positions, shapes, and orientations of the components. Besides, in Fig. 11(b) and Fig. 11 (c), a non-smooth behavior is observed around 300 iterations. The reason for this phenomenon is that during the optimization, the overlapping of the components sometimes leads to a dramatic change in topology, and then results in a high jump in the objective function and volume ratio.

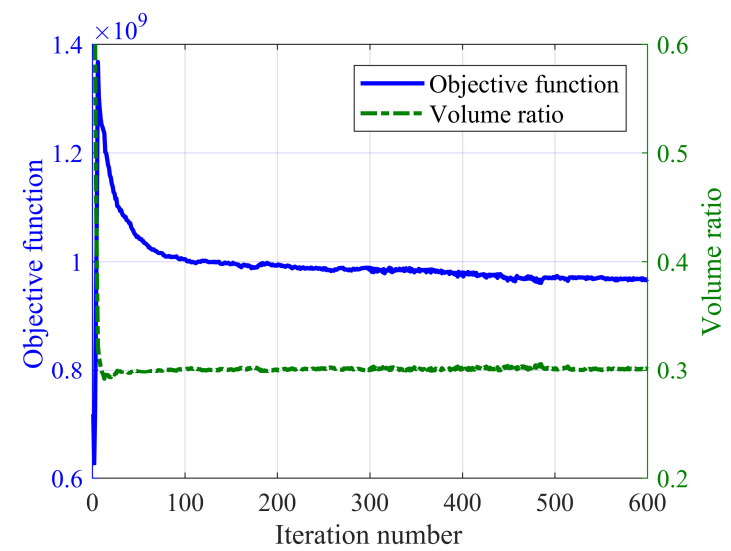

(a)

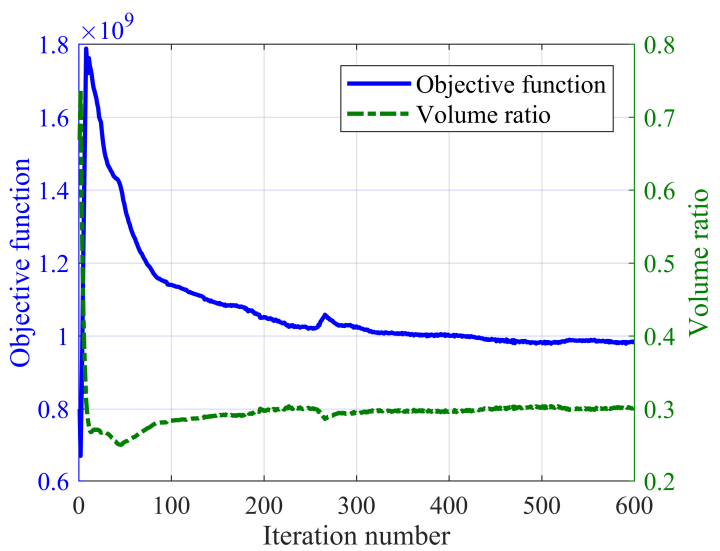

(b)

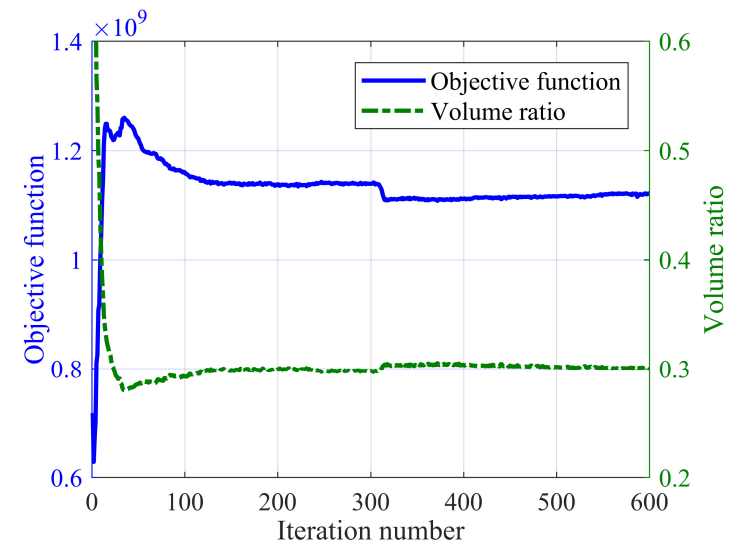

(c)

Fig. 11. Iteration histories of the objective function and volume ratio: (a) non-periodic structure design; (b) heterogeneous periodic structure design; (c) homogeneous periodic structure design.

\subsection{Periodic structure design with different numbers of lattice cells}

In this example, the axially moving structure in Fig. 5 is reemployed and optimized for the periodic structure design with different numbers of lattice cells. Fig. 12(a) and 
Fig. 13(a) present the initial configurations of the components with four and nine lattice cells, respectively. Each lattice cell in Fig. 12(a) and Fig. 13(a) has the same number of components as that in Fig. 9, i.e., 16 components. Therefore, the total numbers of design variables are 576 and 1296 for Fig. 12(a) and Fig. 13(a). To compare with the results with six lattice cells in Fig. 10(b) and (c), both heterogeneous and homogeneous periodic structures are designed for the axially moving structure.

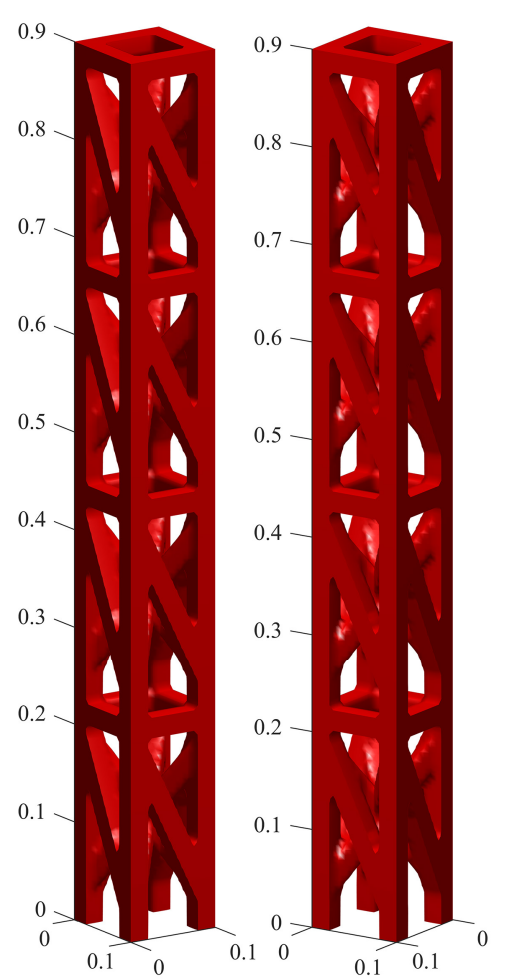

(a)

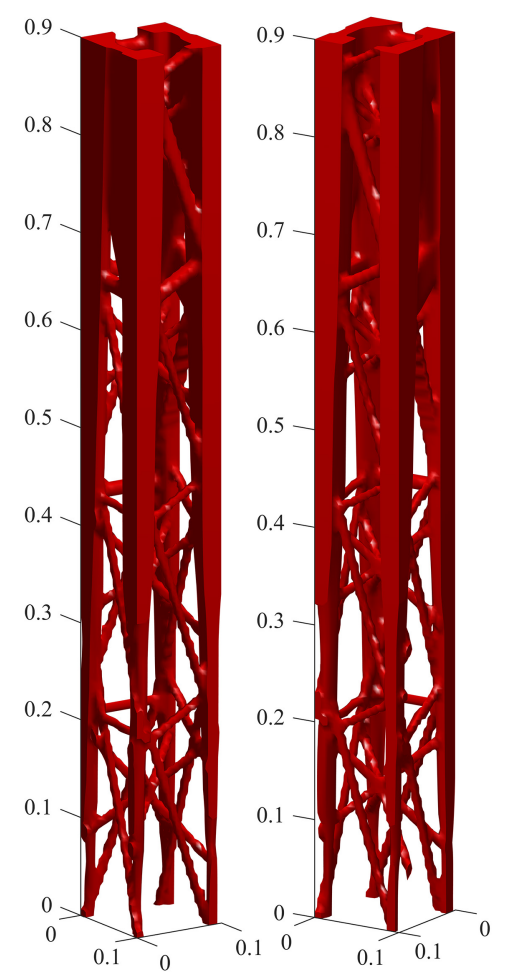

(b)

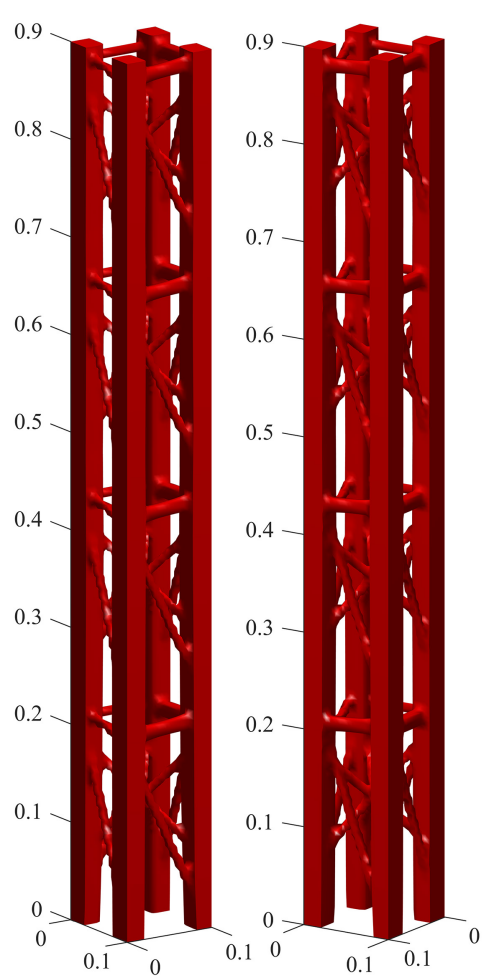

(c)

Fig. 12. Initial configuration and optimized results of the axially moving structure with four lattice cells: (a) initial configuration; (b) heterogeneous periodic structure design; (c) homogeneous periodic structure design.

The results are shown in Fig. 12 and Fig. 13. From these figures, it can be concluded that the simultaneous topology and size optimization approach can deal with the periodic structure design for a 3D variable-length structure with different numbers of lattice cells. The results of the heterogeneous periodic structure design for the axially moving structure in Fig. 12(b) and Fig. 13(b) show the same property as that in Fig. 10(b). They have more material and plate-like components on the top of the axially moving structure. For the results of the homogeneous structure design in Fig. 12(c) and Fig. 13(c), their structures are similar to the result in Fig. 10(c) with four axially thick and strong components on the edges of the structure.

Table 1 Convergent objective function values obtained by using different numbers of lattice cells.

\begin{tabular}{cccc}
\hline Number of lattice cells & 4 & 6 & 9 \\
\hline Heterogeneous periodic structure & $1.0612 \times 10^{9}$ & $0.9832 \times 10^{9}$ & $1.0592 \times 10^{9}$ \\
Homogeneous periodic structure & $1.1544 \times 10^{9}$ & $1.1207 \times 10^{9}$ & $1.1987 \times 10^{9}$ \\
\hline
\end{tabular}




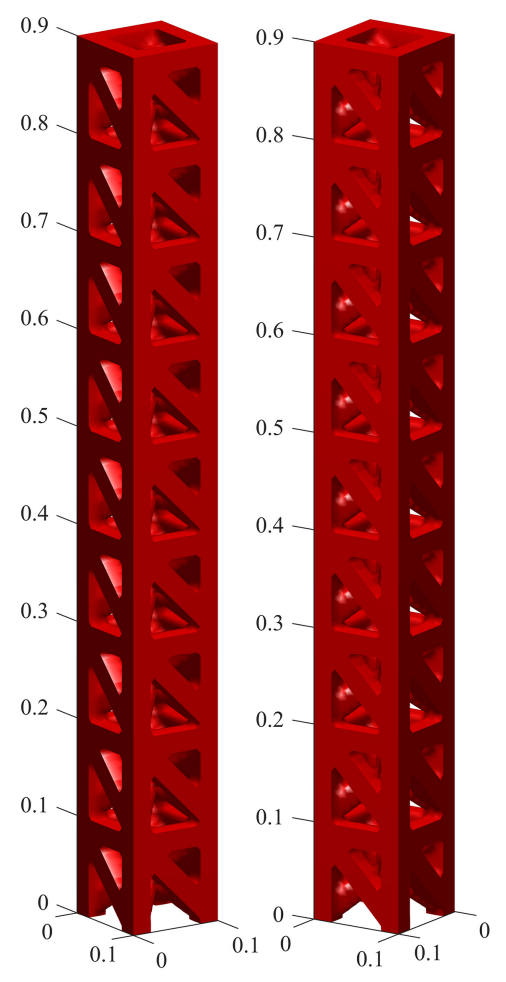

(a)

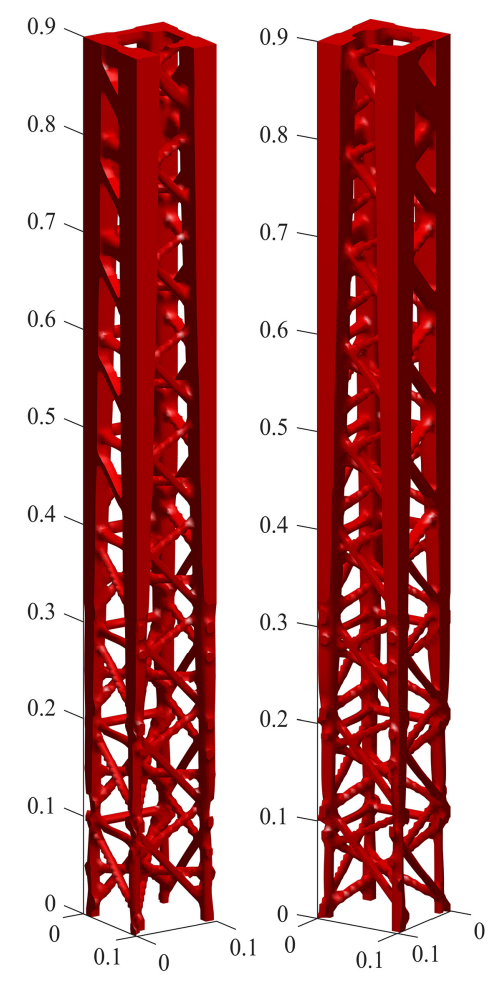

(b)

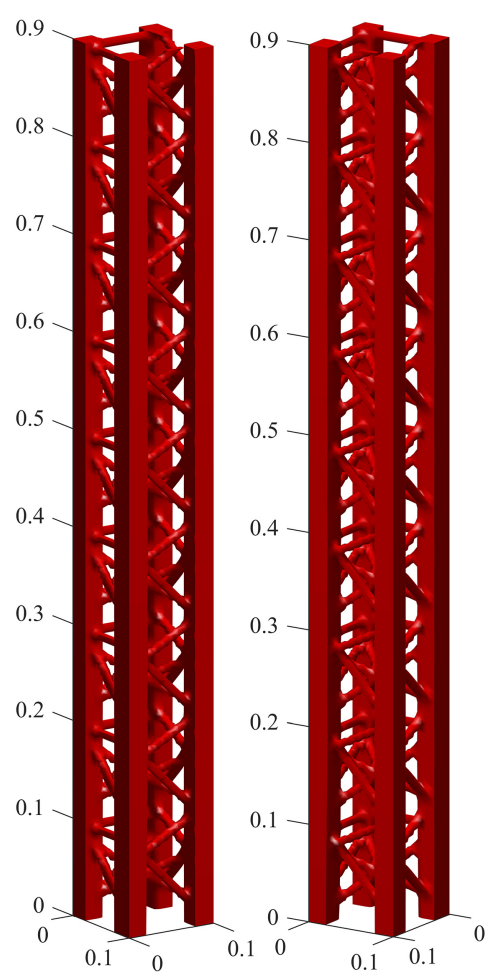

(c)

Fig. 13. Initial configuration and optimized results of the axially moving structure with nine lattice cells: (a) initial configuration; (b) heterogeneous periodic structure design; (c) homogeneous periodic structure design.

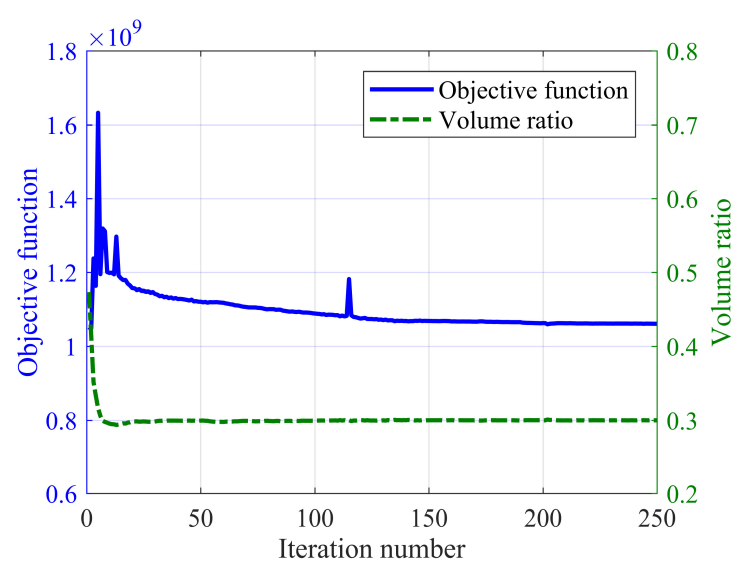

(a)

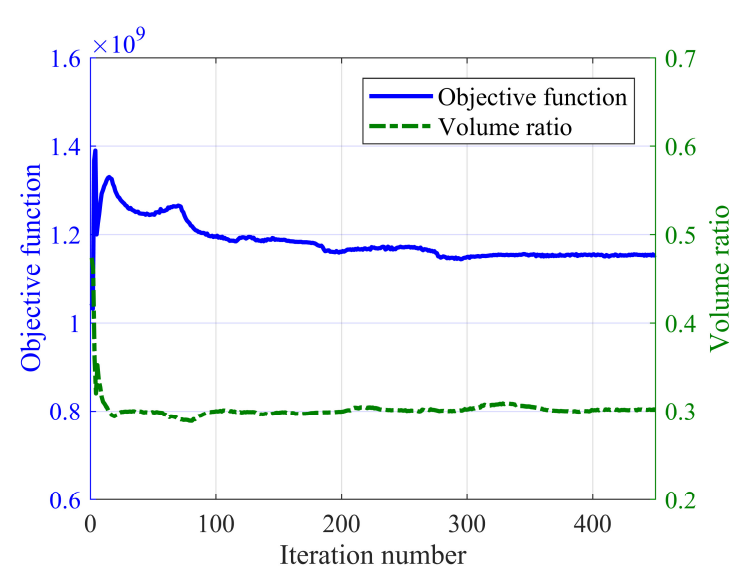

(b)

Fig. 14. Iteration histories of the objective function and volume ratio for the axially moving structure with four lattice cells: (a) heterogeneous periodic structure design; (b) homogeneous periodic structure design.

Fig. 14 and Fig. 15 shows the iteration histories of the objective function and volume ratio for the two cases with different numbers of lattice cells. It can be concluded that the volume constraints are satisfied for all the cases shown in Fig. 12 and Fig. 13. Some oscillations, however, are observed in Fig. 14 and Fig. 15. The reason is that during the optimization, distributed equivalent static loads, rather than a concentrated load, are applied to the structure. Due to the initial configuration of the components, not all the load positions have solid materials. Then, the optimization 
algorithm finds how to change the positions, shapes, and orientations of the components to make sure that most of the high load positions do have solid materials. Some load positions without solid materials, hence, may lead to the oscillations of the design variables, and as a result, the oscillations of the objective function and volume ratio.

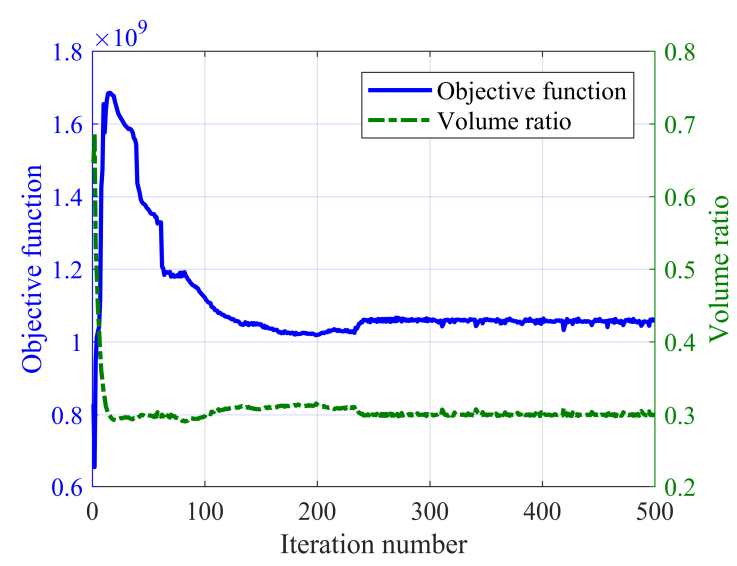

(a)

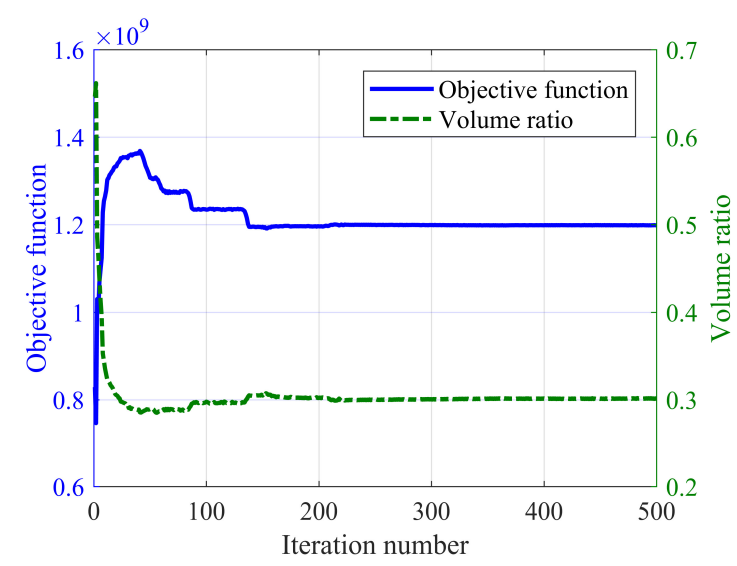

(b)

Fig. 15. Iteration histories of the objective function and volume ratio for the axially moving structure with nine lattice cells: (a) heterogeneous periodic structure design; (b) homogeneous periodic structure design.

From Fig. 14 and Fig. 15, the convergent objective functions of the heterogeneous periodic structure design in Fig. 12(b) and Fig. 13(b) are both smaller than those of the homogeneous periodic structure design in Fig. 12(c) and Fig. 13(c). This conclusion can also be obtained according to Table 1. It can be seen from Table 1 that for the periodic structure design, both heterogeneous and homogeneous, the results for the model with six lattice cells are better than those with four or nine lattice cells. The reason for this phenomenon is that for the models with four or nine lattice cells, the design feasible region is smaller than that with six lattice cells. From Fig. 12(b) and Fig. 12(c), it can be found that for four lattice cells, the separation distances between the components on the four side faces are very large. From Fig. 13(b) and Fig. 13(c), it can be observed that for nine lattice cells, the optimized orientations of the components on the four side faces are very small.

\subsection{Heterogeneous periodic structure design for the deployable structures on a spinning solar sail}

In this example, the simultaneous topology and size optimization approach is further utilized to optimize the deployable structures on a practical spinning solar sail system. As shown in Fig. 16, during the first-stage deployment of a spinning solar sail $[3,52]$, four flexible deployable structures extend from a rigid hub while it rotates and moves along the normal direction of the rotating plane at the same time. Installed at the free end of the four structures, there are four rigid tip masses. The rigid cubic hub has a mass of $400 \mathrm{~kg}$ and an edge length of $1 \mathrm{~m}$. The rigid cubic tip mass has a mass of 5 $\mathrm{kg}$ and an edge length of $0.5 \mathrm{~m}$. The flexible extending structure has an initial length of 
$0.5 \mathrm{~m}$ and a square cross-section of $0.5 \mathrm{~m} \times 0.5 \mathrm{~m}$. The mass density of the structure material is $120 \mathrm{~kg} / \mathrm{m}^{3}$, and Young's modulus and Poison's ratio are $5 \times 10^{8} \mathrm{~Pa}$ and 0.3 , respectively. The gravity is not considered in this example.

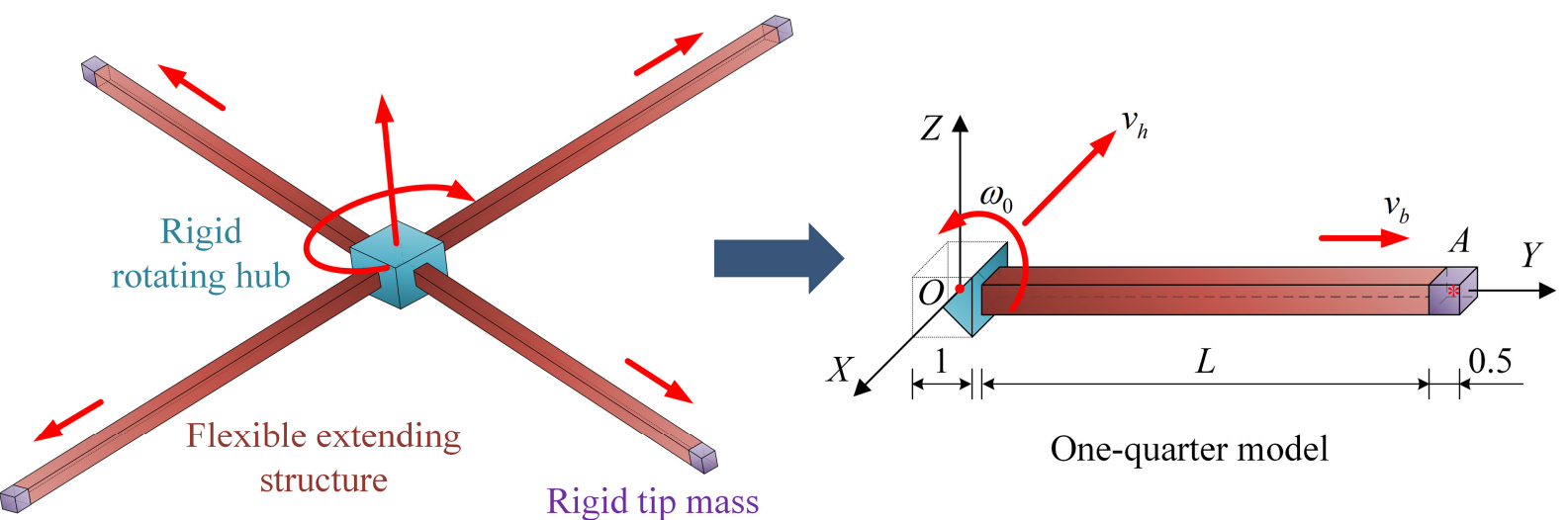

Fig. 16. The first-stage deployment of a spinning solar sail.

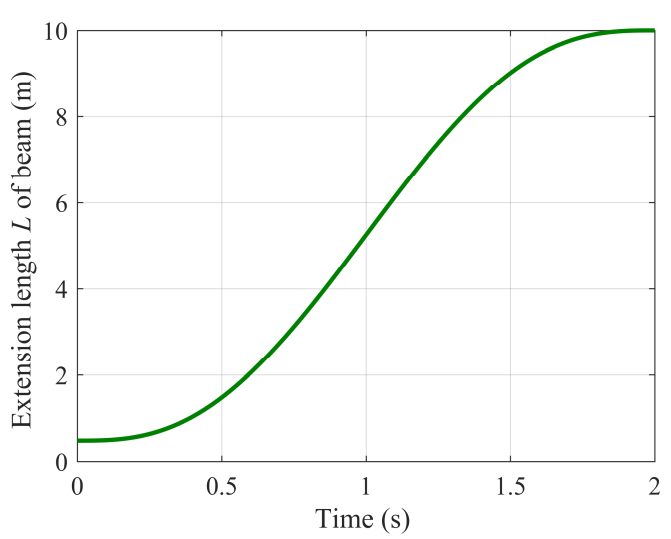

(a)

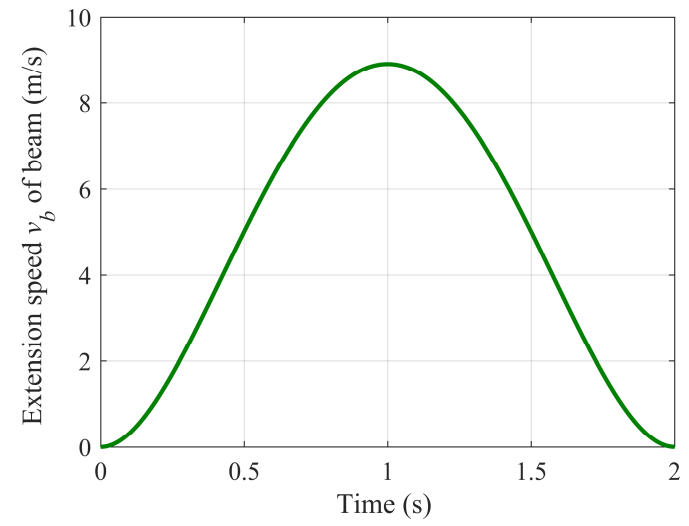

(b)

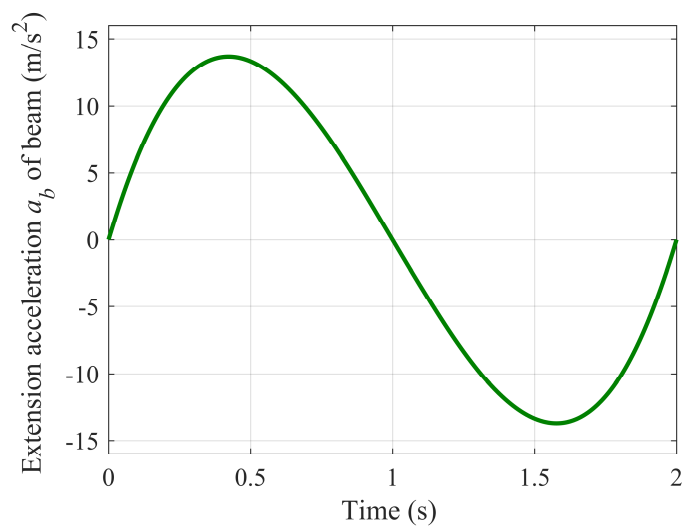

(c)

Fig. 17. The desired extension length, speed and acceleration of the extending structure: (a) extension length $L$; (b) extension speed $v_{b}$; (c) extension acceleration $a_{b}$.

Considering the symmetry of the solar sail system, an one-quarter model is simulated and optimized, as shown in Fig. 16. The one-quarter rigid hub rotates along $X$-axis with an initial angular velocity $\omega_{0}=4 \pi \mathrm{rad} / \mathrm{s}$, and moves along $-X$-axis with a constant speed $v_{h}=5 \mathrm{~m} / \mathrm{s}$. During the deployment, the length of the flexible structure 
extends from $0.5 \mathrm{~m}$ to $10 \mathrm{~m}$ within $2 \mathrm{~s}$. By using a polynomial function of five degrees, the desired extension length $L$ of the structure is shown in Fig. 17(a) to ensure that $L(0)$ $=0.5, L(T)=10, v_{b}(0)=v_{b}(T)=0$ and $a_{b}(0)=a_{b}(T)=0$. The corresponding desired extension speed $v_{b}$ and acceleration $a_{b}$ are then given in Fig. 17(b) and (c).

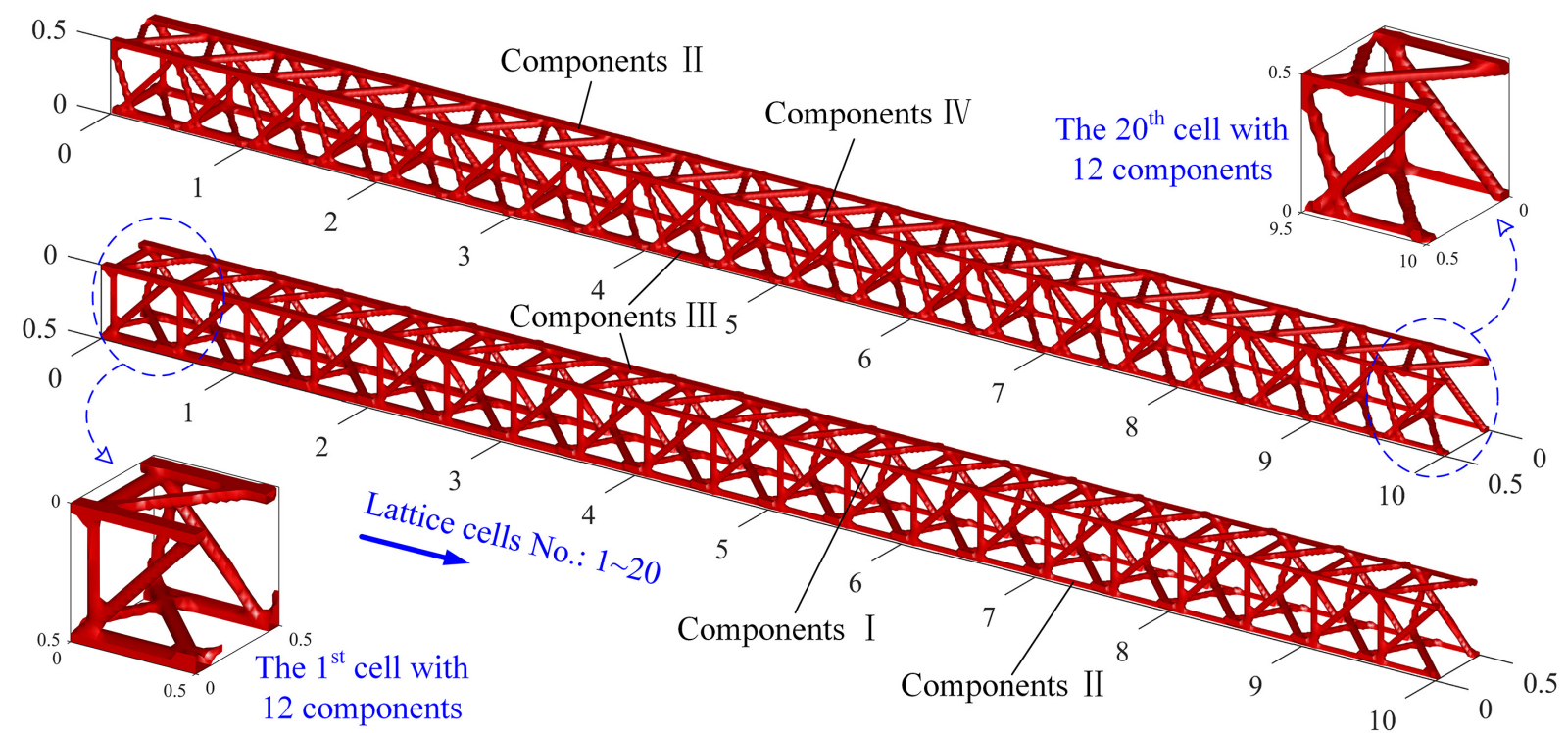

Fig. 18. The optimized result of the heterogeneous periodic structure design for the flexible extending structure.

To optimize the extending structure during the deployment $(t=0 \sim 2 \mathrm{~s})$, the time step size is set as $0.05 \mathrm{~s}$ for ESL analysis so that there are 40 time steps. All the weights are chosen the same for those time steps. For this example, the volume fraction $V_{\max }$ is 0.1 . Fig. 18 presents the optimized result of the heterogeneous periodic structure design for the extending structure in Fig. 16. For a better illustration, Fig. 18 shows the result from two different angles, where both the right ends are the free end of the extending structure. Here, 20 lattice cells are considered and each of them has 12 components. The total number of the design variables is therefore 2160 for this example. The result shown in Fig. 18 is a kind of truss-like structure and obviously has more material on the left part of the extending structure. Table 2 lists the results of the semiheights $L_{3}$ in Eq. (47) for the components I IV. The data in Table 2 tell that the four kinds of components I IV have more thickness in Z-direction on the left part of the extending structure than those on the right. Most of the components on the right part of the extending structure are at the lower boundary of the design variable $L_{3}$, i.e., $0.025 \mathrm{~m}$.

Fig. 19 gives the iteration histories of the objective function and volume ratio for the optimization of the extending structure and shows that the volume constraint $(V \leq 0.1)$ is satisfied. Fig. 20 presents the configurations of the spinning solar sail at four different moments during its first-stage deployment, i.e., $t=0.0,0.5,1.0$ and $2.0 \mathrm{~s}$. Here, it is simply assumed that the four extending structures shown in Fig. 16 will have the same optimized structure to ensure the symmetry of the solar sail system. From Fig. 20, it can be identified that during the deployment of the flexible structures, the rigid hub does rotate and move along the $X$-direction. 


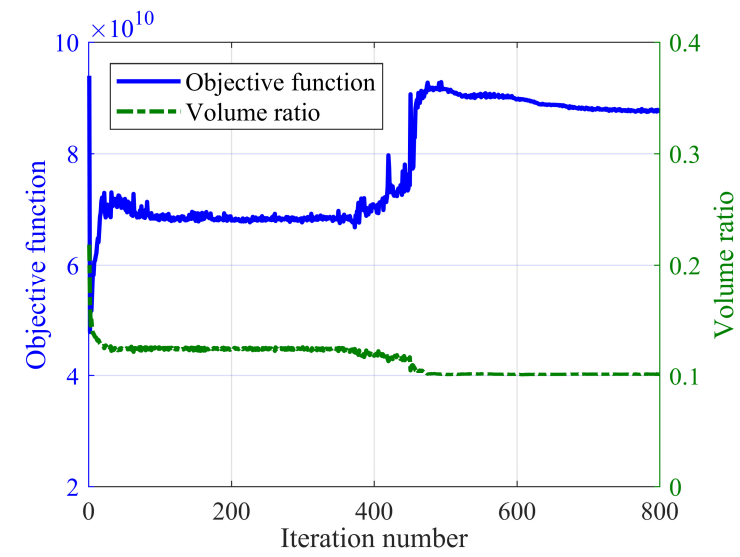

Fig. 19. Iteration histories of the objective function and volume ratio for the flexible extending structure.

Table 2 Optimized semiheights $L_{3}(\mathrm{~m})$ of components I IV for the 20 lattice cells.

\begin{tabular}{ccccc}
\hline Lattice cells No. & Component I & Component II & Component III & Component IV \\
\hline 1 & 0.0430 & 0.0440 & 0.0452 & 0.0431 \\
2 & 0.0432 & 0.0437 & 0.0442 & 0.0432 \\
3 & 0.0433 & 0.0424 & 0.0441 & 0.0441 \\
4 & 0.0431 & 0.0425 & 0.0436 & 0.0438 \\
5 & 0.0433 & 0.0424 & 0.0427 & 0.0430 \\
6 & 0.0431 & 0.0396 & 0.0419 & 0.0430 \\
7 & 0.0430 & 0.0420 & 0.0416 & 0.0429 \\
8 & 0.0434 & 0.0435 & 0.0391 & 0.0425 \\
9 & 0.0405 & 0.0431 & 0.0352 & 0.0401 \\
10 & 0.0365 & 0.0396 & 0.0296 & 0.0368 \\
11 & 0.0316 & 0.0332 & 0.0270 & 0.0292 \\
12 & 0.0302 & 0.0318 & 0.0304 & 0.0296 \\
13 & 0.0303 & 0.0297 & 0.0301 & 0.0302 \\
14 & 0.0250 & 0.0250 & 0.0258 & 0.0250 \\
15 & 0.0250 & 0.0250 & 0.0250 & 0.0250 \\
16 & 0.0250 & 0.0250 & 0.0250 & 0.0250 \\
17 & 0.0250 & 0.0250 & 0.0250 & 0.0250 \\
18 & 0.0250 & 0.0250 & 0.0250 & 0.0250 \\
19 & 0.0250 & 0.0250 & 0.0250 & 0.0250 \\
20 & 0.0250 & 0.0250 & 0.0250 & 0.0250 \\
\hline
\end{tabular}




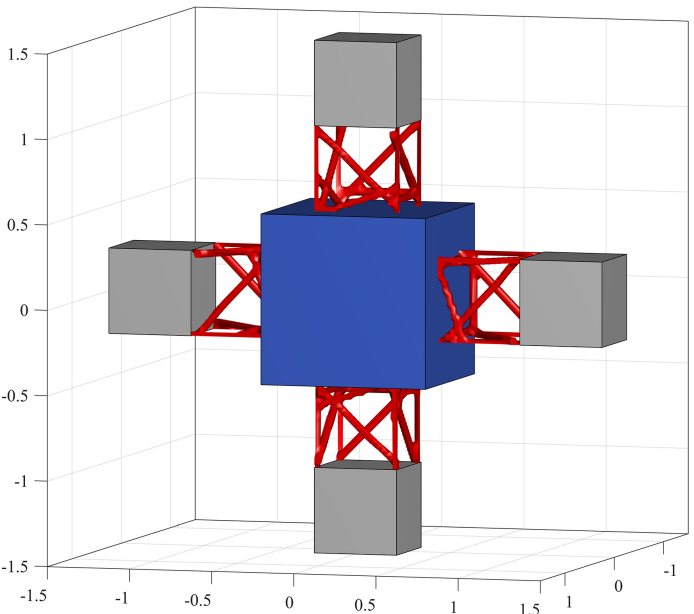

(a)

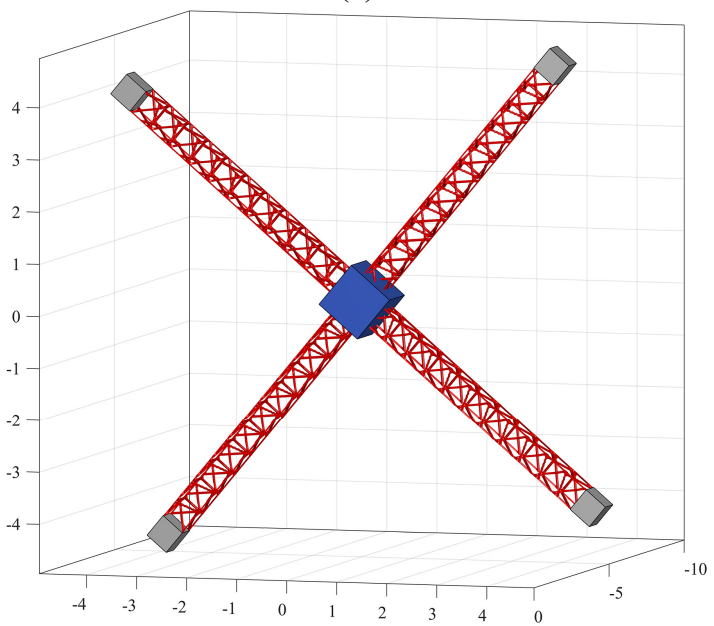

(c)

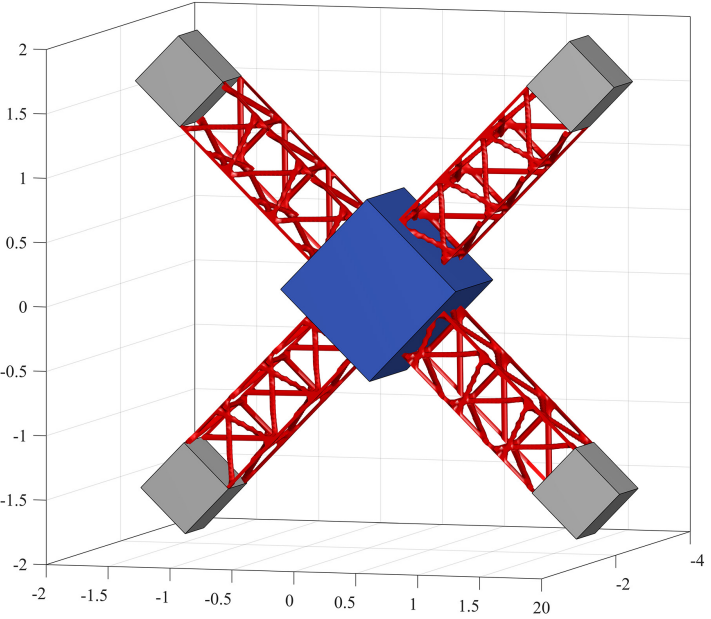

(b)

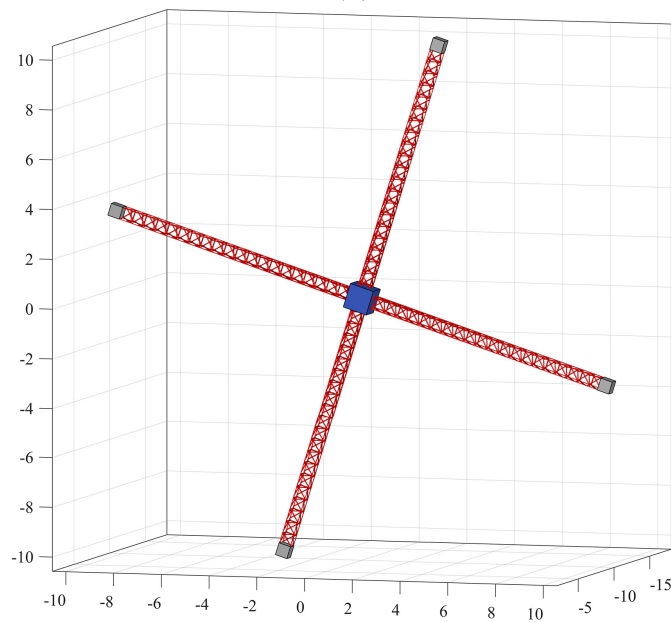

(d)

Fig. 20. Four typical configurations of the optimized spinning solar sail system during its first-stage deployment: (a) $t=0.0 \mathrm{~s}$; (b) $t=0.5 \mathrm{~s}$; (c) $t=1.0 \mathrm{~s}$; (d) $t=2.0 \mathrm{~s}$.

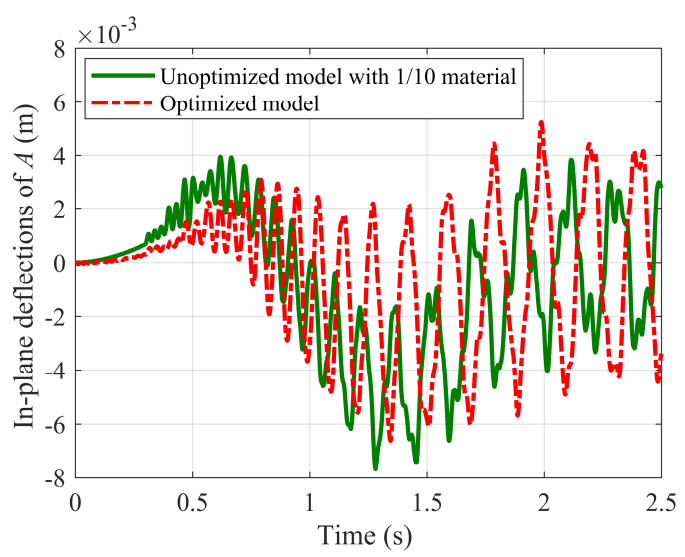

(a)

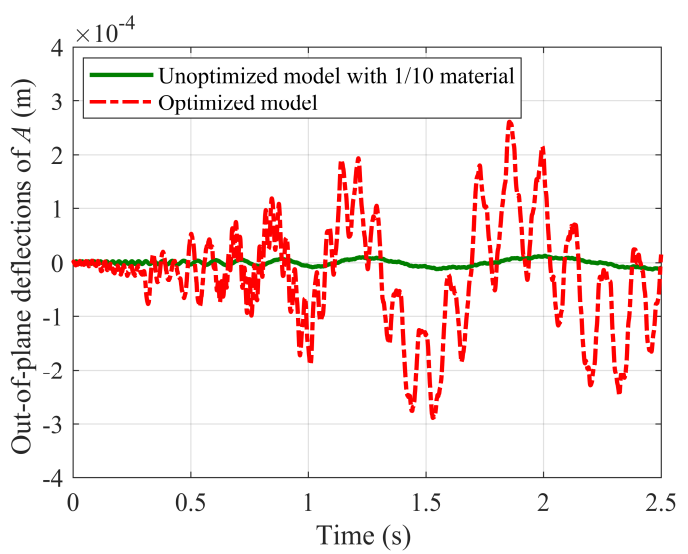

(b)

Fig. 21. Comparisons of the payload centroid $A$ deflections for the unoptimized and optimized models: (a) in-plane deflections of $A$; (b) out-of-plane deflections of $A$.

Fig. 21 compares the deflection responses of the payload centroid $A$ for the unoptimized and optimized models. For a better comparison, the unoptimized model has only $1 / 10$ material which is evenly distributed. Here, the in-plane deflection of point 
$A$ is measured in the rotating plane, that is, YZ-plane in Fig. 16, and the out-of-plane deflection is measured along $X$-direction. For the in-plane deflections of point $A$, the maximum absolute deflection has reduced from $7.689 \times 10^{-3} \mathrm{~m}$ of the unoptimized model to $6.619 \times 10^{-3} \mathrm{~m}$ of the optimized model. It can also be found in Fig. 21(b) that for the unoptimized model, the maximum absolute out-of-plane deflection of point $A$ is $1.251 \times 10^{-5} \mathrm{~m}$, while after optimization the value increases to $2.887 \times 10^{-4} \mathrm{~m}$. Compared with the in-plane deflections in Fig. 21(a), however, the out-of-plane deflections in Fig. 21(b) are far smaller. Therefore, the assertion can still be reached that the optimization of the extending structure is satisfying as the overall stiffness of the structure is actually improved.

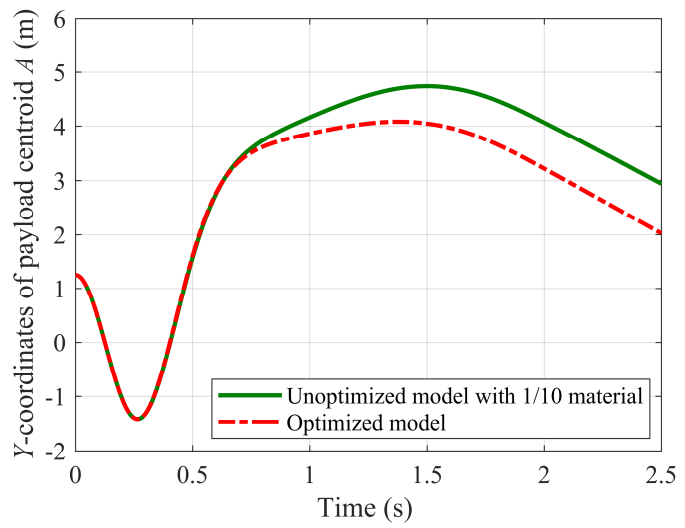

(a)

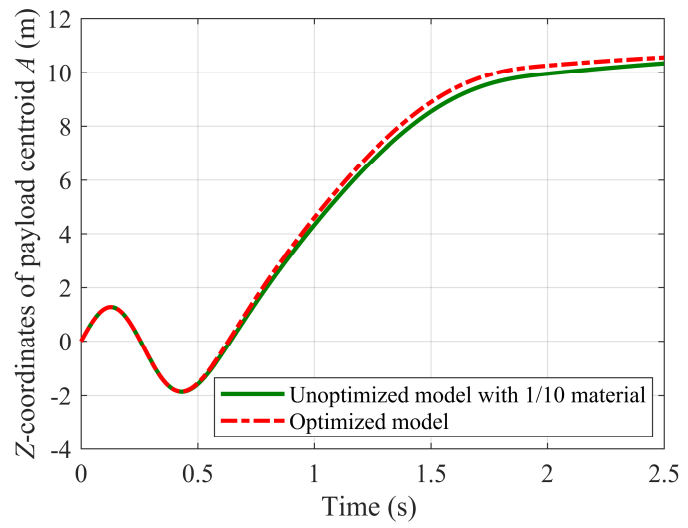

(b)

Fig. 22. Comparisons of the payload centroid $A$ coordinates for the unoptimized and optimized models: (a) $Y$-coordinates of $A$; (b) Z-coordinates of $A$.

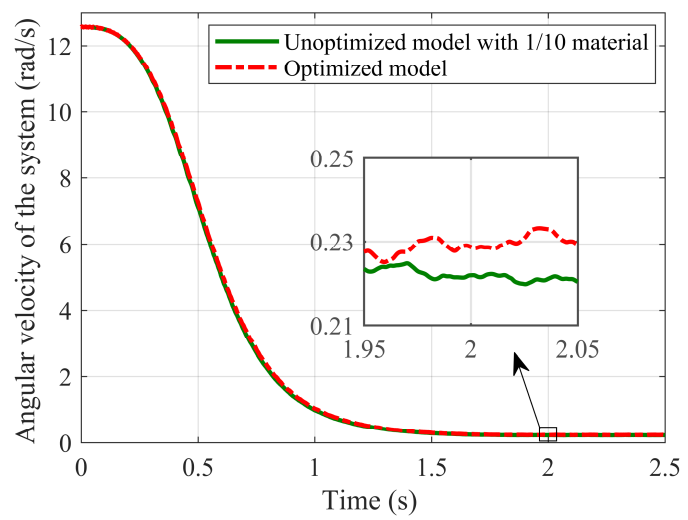

Fig. 23. Comparisons of the system angular velocity for the unoptimized and optimized models.

Fig. 22 presents the comparisons of the point $A$ coordinates for the unoptimized and optimized models and shows that the differences of $Y_{A}$ and $Z_{A}$ between the two models get bigger and bigger with an increase in time. Fig. 22 also indicates that the optimized model rotates a little bit faster than the unoptimized model during the deployment of the flexible extending structures. Fig. 23 straightforwardly reveals that the system angular velocity of the optimized model is slightly greater than that of the unoptimized model when $t=2 \mathrm{~s}$. According to the conservation of angular momentum, with the deployment of the flexible extending structures, the moment of inertial along 
$X$-axis of the whole solar sail system increases. As a consequence, the angular velocity of the system decreases, as clearly shown in Fig. 23. After the optimization, the material distribution is optimized and more material is gathered on the left of the extending structure according to Fig. 18. Under the same desired deployment law in Fig. 17, hence, the moment of inertial along $X$-axis of the optimized model increases more slowly than the unoptimized model with an increase in time.

\section{Concluding remarks}

The paper presents a new simultaneous topology and size optimization approach for a 3D variable-length structure via equivalent static loads (ESL) and moving morphable components (MMC). The ESL analysis employs a flexible multibody system (FMBS) dynamic model for the variable-length structure by using the arbitrary Lagrangian-Eulerian (ALE) formulation and absolute nodal coordinate formulation (ANCF). The developed solid element of ALE-ANCF enables one to accurately describe a 3D FMBS with variable-length bodies undergoing both large overall motions and large deformations. The MMC method uses explicit geometrical parameters, positions, and orientations of a cuboid component as the design variables so as to greatly reduce the number of design variables for the $3 \mathrm{D}$ variable-length structure. By modifying the sensitivities with respect to the linked design variables of different components, the optimization of the variable-length structure can give rise to a homogeneous or a heterogeneous periodic structure. By using the proposed optimization approach, one can simultaneously optimize the topology and thicknesses of all lattice cells and design the variable-length structure as a heterogeneous periodic structure.

The optimization of the axially moving structure shows that the designed heterogeneous periodic structure for the variable-length structure is much better than the designed homogeneous periodic structure, but slightly worse than the designed nonperiodic structure. The optimization of the axially moving structure also gives the comparisons among the designed periodic structures with different numbers of lattice cells at the macro-level. The results reveal that for this specific case, six lattice cells are superior to four or nine lattice cells for the optimization of the axially moving structure. The best number of lattice cells, however, is unknown to the designers but may be determined from the experience of the designers or through a further parametric study. The last example extends the optimization approach to the heterogeneous periodic structure design for a spinning deployable solar sail. The optimized result not only improves the dynamic performance of the deployable structures, but also influences the dynamics of the system such as the angular velocity of the deployment. All the numerical results and analyses indicate that the proposed simultaneous topology and size optimization approach makes it possible to efficiently design a complex 3D FMBS with variable-length bodies. 


\section{Acknowledgments}

This work was supported in part by the National Natural Science Foundation of China under Grants 11521062 and 11722216 and by the 111 China Project (B16003). It was also supported in part by Postgraduate Research \& Practice Innovation Program of Jiangsu Province under Grants KYCX17_0226 and by China Scholarship Council under Grants 201706830011.

\section{Appendix}

The entries of the interpolation function matrix $\mathbf{N}_{e}$ in Eq. (5) are listed as follows

$$
\begin{aligned}
& N_{1,0}=\frac{1}{16}(1+\xi)(1-\eta)(1+\zeta)\left(2+\xi-\eta+\zeta-\xi^{2}-\eta^{2}-\zeta^{2}\right), \\
& N_{1,1}=\frac{1}{32} a\left(\xi^{2}-1\right)(1+\xi)(1-\eta)(1+\zeta), \\
& N_{1,2}=-\frac{1}{32} b\left(\eta^{2}-1\right)(1+\xi)(1-\eta)(1+\zeta), \\
& N_{1,3}=\frac{1}{32} c\left(\zeta^{2}-1\right)(1+\xi)(1-\eta)(1+\zeta), \\
& N_{2,0}=\frac{1}{16}(1-\xi)(1-\eta)(1+\zeta)\left(2-\xi-\eta+\zeta-\xi^{2}-\eta^{2}-\zeta^{2}\right), \\
& N_{2,1}=-\frac{1}{32} a\left(\xi^{2}-1\right)(1-\xi)(1-\eta)(1+\zeta), \\
& N_{2,2}=-\frac{1}{32} b\left(\eta^{2}-1\right)(1-\xi)(1-\eta)(1+\zeta), \\
& N_{2,3}=\frac{1}{32} c\left(\zeta^{2}-1\right)(1-\xi)(1-\eta)(1+\zeta), \\
& N_{3,0}=\frac{1}{16}(1-\xi)(1+\eta)(1+\zeta)\left(2-\xi+\eta+\zeta-\xi^{2}-\eta^{2}-\zeta^{2}\right), \\
& N_{3,1}=-\frac{1}{32} a\left(\xi^{2}-1\right)(1-\xi)(1+\eta)(1+\zeta), \\
& N_{3,2}=\frac{1}{32} b\left(\eta^{2}-1\right)(1-\xi)(1+\eta)(1+\zeta), \\
& N_{3,3}=\frac{1}{32} c\left(\zeta^{2}-1\right)(1-\xi)(1+\eta)(1+\zeta), \\
& N_{4,0}=\frac{1}{16}(1+\xi)(1+\eta)(1+\zeta)\left(2+\xi+\eta+\zeta-\xi^{2}-\eta^{2}-\zeta^{2}\right), \\
& N_{4,2}=\frac{1}{32} a\left(\xi^{2}-1\right)(1+\xi)(1+\eta)(1+\zeta), \\
& \left.N^{2}\right)\left(\eta^{2}-1\right)(1+\xi)(1+\eta)(1+\zeta), \\
& \left.N^{2}\right),
\end{aligned}
$$




$$
\begin{aligned}
& N_{4,3}=\frac{1}{32} c\left(\zeta^{2}-1\right)(1+\xi)(1+\eta)(1+\zeta), \\
& N_{5,0}=\frac{1}{16}(1+\xi)(1-\eta)(1-\zeta)\left(2+\xi-\eta-\zeta-\xi^{2}-\eta^{2}-\zeta^{2}\right) \text {, } \\
& N_{5,1}=\frac{1}{32} a\left(\xi^{2}-1\right)(1+\xi)(1-\eta)(1-\zeta), \\
& N_{5,2}=-\frac{1}{32} b\left(\eta^{2}-1\right)(1+\xi)(1-\eta)(1-\zeta), \\
& N_{5,3}=-\frac{1}{32} c\left(\zeta^{2}-1\right)(1+\xi)(1-\eta)(1-\zeta), \\
& N_{6,0}=\frac{1}{16}(1-\xi)(1-\eta)(1-\zeta)\left(2-\xi-\eta-\zeta-\xi^{2}-\eta^{2}-\zeta^{2}\right), \\
& N_{6,1}=-\frac{1}{32} a\left(\xi^{2}-1\right)(1-\xi)(1-\eta)(1-\zeta) \text {, } \\
& N_{6,2}=-\frac{1}{32} b\left(\eta^{2}-1\right)(1-\xi)(1-\eta)(1-\zeta) \text {, } \\
& N_{6,3}=-\frac{1}{32} c\left(\zeta^{2}-1\right)(1-\xi)(1-\eta)(1-\zeta) \text {, } \\
& N_{7,0}=\frac{1}{16}(1-\xi)(1+\eta)(1-\zeta)\left(2-\xi+\eta-\zeta-\xi^{2}-\eta^{2}-\zeta^{2}\right), \\
& N_{7,1}=-\frac{1}{32} a\left(\xi^{2}-1\right)(1-\xi)(1+\eta)(1-\zeta) \text {, } \\
& N_{7,2}=\frac{1}{32} b\left(\eta^{2}-1\right)(1-\xi)(1+\eta)(1-\zeta), \\
& N_{7,3}=-\frac{1}{32} c\left(\zeta^{2}-1\right)(1-\xi)(1+\eta)(1-\zeta) \text {, } \\
& N_{8,0}=\frac{1}{16}(1+\xi)(1+\eta)(1-\zeta)\left(2+\xi+\eta-\zeta-\xi^{2}-\eta^{2}-\zeta^{2}\right) \text {, } \\
& N_{8,1}=\frac{1}{32} a\left(\xi^{2}-1\right)(1+\xi)(1+\eta)(1-\zeta) \text {, } \\
& N_{8,2}=\frac{1}{32} b\left(\eta^{2}-1\right)(1+\xi)(1+\eta)(1-\zeta), \\
& N_{8,3}=-\frac{1}{32} c\left(\zeta^{2}-1\right)(1+\xi)(1+\eta)(1-\zeta) \text {. }
\end{aligned}
$$

\section{References}

[1] L. Puig, A. Barton, N. Rando, A review on large deployable structures for astrophysics missions, Acta Astronautica 67 (2010) 12-26.

[2] Y. Peng, Z. Zhao, M. Zhou, J. He, J. Yang, Flexible multibody model and the dynamics of the deployment of mesh antennas, Journal of Guidance, Control, and Dynamics 40(6) (2017) 14991510.

[3] J. Sun, Q. Tian, H. Hu, N.L. Pedersen, Topology optimization of a flexible multibody system with 
variable-length bodies described by ALE-ANCF, Nonlinear Dynamics 93(2) (2018) 413-441.

[4] D. Gross, D. Messner, The able deployable articulated mast-enabling technology for the shuttle radar topography mission. in Proceedings of the 33rd Aerospace Mechanisms Symposium, May 19-21, 1999, Pasadena, California.

[5] Y. Tang, A. Kurtz, Y.F. Zhao, Bidirectional Evolutionary Structural Optimization (BESO) based design method for lattice structure to be fabricated by additive manufacturing, Computer-Aided Design 69 (2015) 91-101.

[6] H. Hayashi, S. Takehara, Y. Terumichi, Numerical approach for flexible body motion with large displacement and Time-Varying length. The 3rd Joint International Conference on Multibody System Dynamics and the 7th Asian Conference on Multibody Dynamics, June 30-July 3, 2014, BEXCO, Busan, Korea.

[7] Y. Terumichi, S. Kaczmarczyk, K. Sogabe, Numerical approach in the analysis of flexible body motion with Time-Varying length and large displacement using multiple time scales. The 1st Joint International Conference on Multibody System Dynamics, May 25-27, 2010, Lappeenranta, Finland.

[8] D.F. Hong, G.X. Ren, A modeling of sliding joint on one-dimensional flexible medium, Multibody System Dynamics 26(1) (2011) 91-106.

[9] P. Hyldahl, A. Mikkola, O. Balling, A thin plate element based on the combined arbitrary Lagrange-Euler and absolute nodal coordinate formulations, Proceedings of the Institution of Mechanical Engineers, Part K: Journal of Multi-Body Dynamics 227(3) (2013) 211-219.

[10] M. Géradin, A. Cardona, Flexible multibody dynamics: A finite element approach, John Wiley \& Sons, New York, 2001.

[11] D.F. Hong, J.L. Tang, G.X. Ren, Dynamic modeling of mass-flowing linear medium with large amplitude displacement and rotation, Journal of Fluids and Structures 27(8) (2011) 1137-1148.

[12] J.L. Tang, G.X. Ren, W.D. Zhu, H. Ren, Dynamics of variable-length tethers with application to tethered satellite deployment, Communications in Nonlinear Science and Numerical Simulation 16(8) (2011) 3411-3424.

[13] J.L. Escalona, An arbitrary Lagrangian-Eulerian discretization method for modeling and simulation of reeving systems in multibody dynamics, Mechanism and Machine Theory 112 (2017) $1-21$.

[14] A. Pechstein, J. Gerstmayr, A Lagrange-Eulerian formulation of an axially moving beam based on the absolute nodal coordinate formulation, Multibody System Dynamics 30(3) (2013) 343-358.

[15] J.L. Sun, Q. Tian, H.Y. Hu, Structural optimization of flexible components in a flexible multibody system modeled via ANCF, Mechanism and Machine Theory 104 (2016) 59-80.

[16] E. Tromme, A. Held, P. Duysinx, O. Brüls, System-Based approaches for structural optimization of flexible mechanisms, Archives of Computational Methods in Engineering 25(3) (2018) 817-844.

[17] E. Tromme, D. Tortorelli, O. Brüls, P. Duysinx, Structural optimization of multibody system components described using level set techniques, Structural and Multidisciplinary Optimization 52(5) (2015) 959-971.

[18] E. Tromme, O. Brüls, P. Duysinx, Weakly and fully coupled methods for structural optimization of flexible mechanisms, Multibody System Dynamics 38(4) (2016) 391-417.

[19] J.L. Sun, Q. Tian, H.Y. Hu, Topology optimization based on level set for a flexible multibody system modeled via ANCF, Structural and Multidisciplinary Optimization 55 (2017) 1159-1177.

[20] E. Tromme, V. Sonneville, J.K. Guest, O. Brüls, System-wise equivalent static loads for the design 
of flexible mechanisms, Computer Methods in Applied Mechanics and Engineering 329 (2018) 312-331.

[21] E. Tromme, V. Sonneville, O. Brüls, P. Duysinx, On the equivalent static load method for flexible multibody systems described with a nonlinear finite element formalism, International Journal for Numerical Methods in Engineering 108(6) (2016) 646-664.

[22] A. Moghadasi, A. Held, R. Seifried, Modeling of revolute joints in topology optimization of flexible multibody systems, Journal of Computational and Nonlinear Dynamics 12(1) (2017) 011015.

[23] J.L. Sun, Q. Tian, H.Y. Hu, Topology optimization of a three-dimensional flexible multibody system via moving morphable components, Journal of Computational and Nonlinear Dynamics 13(2) (2018) 021010.

[24] E.P. Hong, B.J. You, C.H. Kim, G.J. Park, Optimization of flexible components of multibody systems via equivalent static loads, Structural and Multidisciplinary Optimization 40(1-6) (2010) 549-562.

[25] B.S. Kang, G.J. Park, J.S. Arora, Optimization of flexible multibody dynamic systems using the equivalent static load method, AIAA Journal 43(4) (2005) 846-852.

[26] G. Reinhart, S. Teufelhart, Load-Adapted design of generative manufactured lattice structures, Physics Procedia 12 (2011) 385-392.

[27] X.J. Gu, J.H. Zhu, W.H. Zhang, The lattice structure configuration design for stereolithography investment casting pattern using topology optimization, Rapid Prototyping Journal 18(5) (2012) 353-361.

[28] T. Stankovic, J. Mueller, P. Egan, K. Shea, A generalized optimality criteria method for optimization of additively manufactured multimaterial lattice structures, Journal of Mechanical Design 137(11) (2015) 111705.

[29] P. Zhang, J. Toman, Y. Yu, E. Biyikli, M. Kirca, M. Chmielus, A.C. To, Efficient DesignOptimization of Variable-Density hexagonal cellular structure by additive manufacturing: Theory, Journal of Manufacturing Science and Engineering 137(2) (2015) 021004.

[30] C. Liu, Z.L. Du, W.S. Zhang, Y.C. Zhu, X. Guo, Additive Manufacturing-Oriented design of graded lattice structures through explicit topology optimization, Journal of Applied Mechanics 84(8) (2017) 081008.

[31] T. Wu, K. Liu, A. Tovar, Multiphase topology optimization of lattice injection molds, Computers and Structures 192 (2017) 71-82.

[32] Y. Wang, L. Zhang, S. Daynes, H. Zhang, S. Feih, M.Y. Wang, Design of graded lattice structure with optimized mesostructures for additive manufacturing, Materials \& Design 142 (2018) 114123.

[33] Y. Wang, F. Chen, M.Y. Wang, Concurrent design with connectable graded microstructures, Computer Methods in Applied Mechanics and Engineering 317 (2017) 84-101

[34] A. Asadpoure, J.K. Guest, L. Valdevit, Incorporating fabrication cost into topology optimization of discrete structures and lattices, Structural and Multidisciplinary Optimization 51(2) (2015) 385396.

[35] J. Nguyen, S.I. Park, D. Rosen, Heuristic optimization method for cellular structure design of light weight components, International Journal of Precision Engineering and Manufacturing 6(14) (2013) 1071-1078.

[36] S. Teufelhart, Investigation of the capability of flux of force oriented lattice structures for 
lightweight design, Advanced Materials Research 907 (2014) 75-87.

[37] X. Guo, W.S. Zhang, W.L. Zhong, Doing topology optimization explicitly and geometrically-a new moving morphable components based framework, Journal of Applied Mechanics 81(8) (2014) 081009.

[38] X. Guo, W.S. Zhang, J. Zhang, J. Yuan, Explicit structural topology optimization based on moving morphable components (MMC) with curved skeletons, Computer Methods in Applied Mechanics and Engineering 310 (2016) 711-748.

[39] W.S. Zhang, D. Li, J. Yuan, J.F. Song, X. Guo, A new three-dimensional topology optimization method based on moving morphable components (MMCs), Computational Mechanics 69(4) (2017) 647-665.

[40] W.S. Zhang, J. Yuan, J. Zhang, X. Guo, A new topology optimization approach based on Moving Morphable Components (MMC) and the ersatz material model, Structural and Multidisciplinary Optimization 53(6) (2016) 1243-1260.

[41] H. Goldstein, C. Poole, J. Safko, Classical mechanics, Pearson Education, Inc.16-21, 2002.

[42] J. Chung, G.M. Hulbert, A time integration algorithm for structural dynamics with improved numerical dissipation: The generalized $\alpha$-method, Journal of Applied Mechanics 60(2) (1993) 371375.

[43] M. Arnold, O. Brüls, Convergence of the generalized- $\alpha$ scheme for constrained mechanical systems, Multibody System Dynamics 18 (2007) 185-202.

[44] Q. Tian, P. Flores, H.M. Lankarani, A comprehensive survey of the analytical, numerical and experimental methodologies for dynamics of multibody mechanical systems with clearance or imperfect joints, Mechanism and Machine Theory 122 (2018) 1-57.

[45] V.A. Leontiev, Extension of LMS formulations for L-stable optimal integration methods with U0 V0 overshoot properties in structural dynamics: The level-symmetric (LS) integration methods, International Journal for Numerical Methods in Engineering 71(13) (2007) 1598-1632.

[46] K.K. Tamma, J. Har, X. Zhou, M. Shimada, A. Hoitink, An overview and recent advances in vector and scalar formalisms: Space/Time discretizations in computational dynamics - a unified approach, Archives of Computational Methods in Engineering 18(2) (2011) 119-283.

[47] H.P. Shao, The studying on the direct time integration algorithms for structural dynamics response. 1987, Zhejiang University.

[48] H.P. Shao, C.W. Cai, A three parameters algorithm for numerical integration of structural dynamic equations, Chinese Journal of Applied Mechanics 5(4) (1988) 76-81.

[49] H.A. Lee, G.J. Park, Nonlinear dynamic response topology optimization using the equivalent static loads method, Computer Methods in Applied Mechanics and Engineering 283 (2015) 956-970.

[50] H.H. Jang, H.A. Lee, J.Y. Lee, G.J. Park, Dynamic response topology optimization in the time domain using equivalent static loads, AIAA Journal 50(1) (2012) 226-234.

[51] K. Svanberg, The method of moving asymptotes - a new method for structural optimization, International Journal for Numerical Methods in Engineering 24(2) (1987) 359-373.

[52] H. Sakamoto, Y. Miyazaki, O. Mori, Transient dynamic analysis of gossamer appendage deployment using nonlinear finite element method, Journal of Spacecraft and Rockets 48(5) (2011) 881-890. 